\title{
Topology optimization of multi-scale structures: a review
}

\author{
Jun $\mathrm{Wu}^{1}$ D . Ole Sigmund ${ }^{2}$. Jeroen P. Groen ${ }^{2}$
}

Received: 23 December 2020 / Revised: 5 February 2021 / Accepted: 11 February 2021 / Published online: 8 March 2021 (C) The Author(s) 2021

\begin{abstract}
Multi-scale structures, as found in nature (e.g., bone and bamboo), hold the promise of achieving superior performance while being intrinsically lightweight, robust, and multi-functional. Recent years have seen a rapid development in topology optimization approaches for designing multi-scale structures, but the field actually dates back to the seminal paper by Bendsøe and Kikuchi from 1988 (Computer Methods in Applied Mechanics and Engineering 71(2): pp. 197-224). In this review, we intend to categorize existing approaches, explain the principles of each category, analyze their strengths and applicabilities, and discuss open research questions. The review and associated analyses will hopefully form a basis for future research and development in this exciting field.
\end{abstract}

Keywords Topology optimization $\cdot$ Multi-scale structures $\cdot$ Multi-scale modelling $\cdot$ Additive manufacturing

\section{Introduction}

Topology optimization is a computational design method for automatically generating a structural layout with maximized performance under relevant design specifications. In other words, the structural design problem can be formulated as optimizing the distribution of material in a discretized design domain (Bendsøe 1989). The optimized layout is not restricted to its initial topology, opening for superior structural performance over manual designs based on engineers' intuition and experience. This capability is especially attractive and has been successfully applied in high-tech industries, such as aerospace (Zhu et al. 2016), automotive (Yang and Chahande 1995), architecture (Beghini et al. 2014), and healthcare (Wang et al. 2016).

Early works that lead to the establishment of this field, and in particular the seminal work by Bendsøe and Kikuchi (1988), made use of a material model corresponding

Responsible Editor: Ming Zhou

Jun Wu

j.wu-1@tudelft.nl

1 Department of Sustainable Design Engineering, Delft University of Technology, Landbergstraat 15, 2628 CE, Delft, The Netherlands

2 Department of Mechanical Engineering, Technical University of Denmark, Nils Koppels Allé, B.404, 2800, Kgs. Lyngby, Denmark to infinitely small square cells with rectangular holes and evaluated equivalent mechanical properties of these cells by numerical homogenization. Theoretically, optimal structures comprise spatially varying geometric patterns spanning multiple length scales. Due to manufacturing difficulties of these multi-scale structures, focus in the late 1990s shifted from homogenization-based approaches to "mono-scale" approaches, optimizing the distribution of a homogeneous isotropic material (Bendsøe 1989; Zhou and Rozvany 1991; Mlejnek 1992; Bendsøe and Sigmund 1999). Popular methods such as those based on density (Sigmund 2001), level sets (Wang et al. 2003; Allaire et al. 2004), and evolutionary procedures (Xie and Steven 1993) all belong to this latter category.

Over the past few years, along with advances in additive manufacturing (AM, also known as 3D printing), there has been a resurgent interest in optimal design of multi-scale structures. In additive manufacturing, parts are produced layer upon layer by, e.g., extruding small flattened strings of molten material or melting and fusing powder material or wire. AM provides an effective means to fabricate complex mono-scale structures as well as delicate multiscale structures. In fact, topology optimization of (monoscale) structures and the use of lattice infill are recognized as two dominating strategies for designing next-generation lightweight structures (Brackett et al. 2011; Thompson et al. 2016; Plocher and Panesar 2019). The combination, i.e., topology optimization of multi-scale structures, thus holds the promise of superior performance in a general sense. 
Fig. 1 Illustration of a multi-scale structure

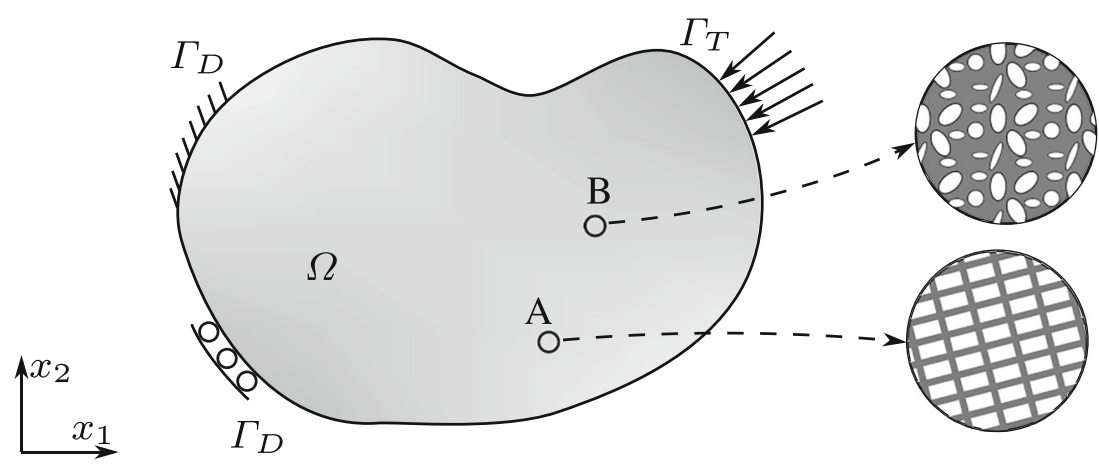

These expectations are sometimes coupled with observation of multi-scale structures found in nature (e.g., bone and bamboo (Lakes 1993; Fratzl and Weinkamer 2007)), while the specific function and the driving mechanisms underlying those natural multi-scale structures are not always known precisely.

Figure 1 illustrates a multi-scale structure. Each point in the macroscale structure effectively represents a periodic repetition of a local microstructure. Here, as common in the topology optimization literature, microstructure shall be interpreted relatively-it does not specifically refer to a physical size under, e.g., $1 \mathrm{~mm}$ but rather to a scale much smaller than the macroscale. It is thus assumed that continuum mechanics is applicable to both macro- and microscales. Some examples of 3D multi-scale structures designed by topology optimization are shown in Fig. 2 .

In view of the rapid development of topology optimization of multi-scale structures, it seems timely to critically review the variety of methods that have been proposed. The term multi-scale has been used extensively in the literature (and also in this review) to describe structures and modelling techniques, as well as design approaches. Many design approaches make use of multi-scale modelling, i.e., they assume separation of length scales. However, monoscale modelling can also be used to design multi-scale structures. In this review, we intend to categorize existing approaches, explain the principles of each category, analyze their strengths and applicabilities, and discuss open research questions. This review is supplemented with our implementation of representative approaches, based on which we quantitatively evaluate structural performance. This review and analysis hopefully will form a basis for future research and development in this exciting field.

\section{Background}

In this section, we categorize three fundamental formulations that provide the basis for most multi-scale approaches.

\subsection{Homogenization-based structural optimization}

A starting point in the history of structural optimization is the classical work by Michell (1904) where an optimal truss design is represented by a continuum description. Arguably, this is the first work on multi-scale optimization since the truss-like continuum description is actually a limit case that can only be realized by using an infinite number of discrete truss members; see Prager and Rozvany (1977) for more details. Similarly, Cheng and Olhoff
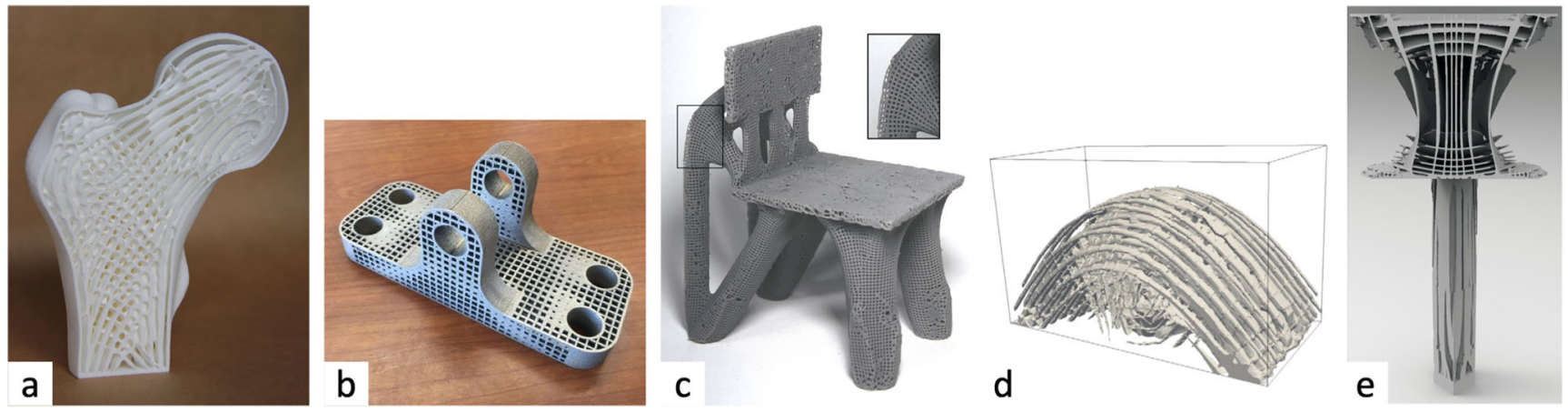

Fig. 2 Some examples of 3D multi-scale structures designed by topology optimization. From left to right: a bone-inspired infill structures, b variable-density lattice optimization, and (c,d,e) three dehomogenization schemes in 3D. The first three are produced by $3 \mathrm{D}$ printing, while the last two are renderings. a and c Reprinted with permission from IEEE, from Wu et al. (2018) and Wu et al. (2021), respectively. b, $\mathbf{d}$ and $\mathbf{e}$ Reprinted with permission from Elsevier, from Zhang et al. (2017), Geoffroy-Donders et al. (2020)and Groen et al. (2020), respectively 
Fig. 3 Layout of the unit cell with a rectangular hole, in local $\left(y_{1}, y_{2}\right)$, and global $\left(x_{1}, x_{2}\right)$ coordinate systems
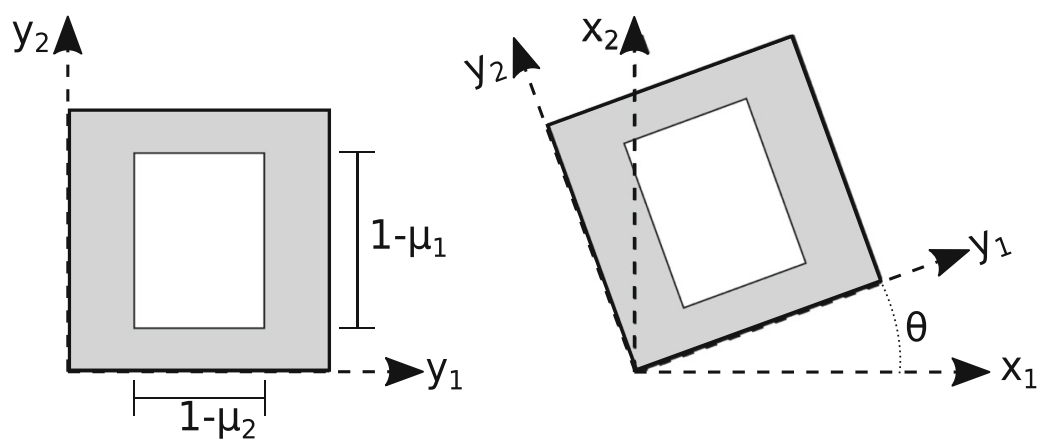

(1981) found that for the case of optimizing the stiffness of a plate by varying the thickness, more stiffener-like reinforcement appeared when the design space was refined. They made the important conclusion that in the limit of an infinitely fine mesh an infinite number of stiffener-like reinforcing members would occur (Cheng 1981). Only by restricting the variation in shape can existence of a solution be proven (Niordson 1983). For compliance minimization in 2 or 3 dimensions, Kohn and Strang (1986) proved that the optimal material distribution can only be found by relaxing the design space. This means the use of a continuous composite material description using a characteristic unit-cell length $\epsilon \rightarrow 0$, allowing for much more detail than a single-scale discretized point-wise material or void description. Here, the theory of homogenization (Bensoussan et al. 1978) enters the field of structural optimization, since this is the perfect tool to bridge the scale between the microscopic periodic composite material and its effective (homogenized) properties on the macroscale. Using this theory, several research groups independently and more or less simultaneously realized that there exists a class of sequential laminates, the so-called rank- $N$ laminates that can achieve the theoretical upper bounds for maximum strain energy density (Lurie and Cherkaev 1984; Norris 1985; Francfort and Murat 1986; Milton 1986). These composite materials consist of several scales in themselves, which is a necessary condition to achieve ultimate stiffness (Allaire and Aubry 1999). With this knowledge, Bendsøe and Kikuchi (1988) came up with the landmark paper that provided the computational framework to do homogenization-based topology optimization of continuum structures. Contrary to using rank-2 laminates that are optimal for a single load case in $2 \mathrm{D}$, they came up with a single-scale interpretation, the square microstructure with a rectangular hole, shown in Fig. 3. By changing the widths $\mu_{1}$ and $\mu_{2}$ as well as the orientation $\theta$ throughout the design domain, near-optimal structures could be achieved. The use of rank-2 laminates as a microstructure was included in later works (Bendsøe 1989; Allaire and Kohn 1993).

\subsection{Density-based topology optimization}

Shortly after the homogenization-based topology optimization approach was introduced, an alternative known as the SIMP (Solid Isotropic Material with Penalization) or powerlaw approach was suggested (Bendsøe 1989; Zhou and Rozvany 1991; Mlejnek 1992). Here, the material distribution in a design domain is represented by a scalar field, with a relative density per element in the discretized domain. Here, $\rho=1$ means solid and $\rho=0$ means empty. This integer-programming problem is relaxed to allow for intermediate densities during optimization. Regardless of the value of the relative density, the material within each element is assumed to be isotropic and homogeneous. Material properties (e.g., Young's modulus) are related to relative densities by a power-law interpolation. This seemingly artificial relationship was later proved physically permissible for a properly chosen penalty power, e.g., with a power $p \geq 3$ for Poisson's ratio $v=1 / 3$ (Bendsøe and Sigmund 1999). Based on this, a heterogeneous material distribution within the element domain, i.e., a microstructure of solid base material and void, can be found to match the expected material properties of an intermediate density. Hence, even the simple "mono-scale" density approaches may be interpreted as multi-scale approaches albeit with suboptimal microstructures. With a justified penalization power, the SIMP approach converges to near 0-1 solutions, representing mono-scale structures. In a number of multi-scale approaches to be discussed later, a smaller $p$ is chosen for obtaining solutions with a large portion of intermediate densities, which provide a basis for filling porous microstructures. However, this may not be mechanically valid, c.f. if $p<3$ for $v=1 / 3$, this power-law scheme violates the Hashin-Strikhman bounds (Hashin and Shtrikman 1963). The SIMP approach is easy to implement; see educational codes (Sigmund 2001; Andreassen et al. 2011; Ferrari and Sigmund 2020). Here, the three-field projection approach is recommended (Guest et al. 2004; Xu et al. 2010; Wang et al. 2011). 
Mono-scale structures can also be optimized by methods based on level sets (Wang et al. 2003; Allaire et al. 2004), evolutionary procedures (Xie and Steven 1993), and geometric morphing and projection (Norato et al. 2004; Guo et al. 2014). For an overview of these methods, we refer to the reviews by Sigmund and Maute (2013) and Deaton and Grandhi (2014). The use of non-gradient approaches for solving topology optimization problems with many variables is not recommended (Sigmund 2011).

\subsection{Inverse homogenization}

The theory of numerical homogenization, which approximates effective properties of a periodic unit cell, can also be exploited to design a microstructure with desired properties. This method was introduced by Sigmund (1994) and is generally referred to as inverse homogenization. With compliance minimization in mind, it is common practice to formulate an optimization problem to maximize the microstructure stiffness with respect to the applied stresses or strains. For the difference in stiffness between such microstructures and the optimal energy bounds, see, e.g., Guedes et al. (2003) and Träff et al. (2019). Furthermore, microstructures with negative Poisson's ratio (Sigmund 1994; Andreassen et al. 2014; Clausen et al. 2015b), materials with maximum shear and bulk moduli (Sigmund 2000), or materials with increased buckling strength (Thomsen et al. 2018; Wang and Sigmund 2020) can be designed using inverse homogenization.

Besides problems in elasticity, inverse homogenization can be applied to many other types of physics. Sigmund and Torquato (1996) considered thermal expansion problems, while Torquato et al. (2002) included both thermal and electrical conductivity. Sigmund (1999) and Challis et al. (2012) considered combined stiffness and fluid permeability. Inverse homogenization for the design of phononic and photonic materials has been considered by Sigmund and Jensen (2003) and Jensen and Sigmund (2011), respectively. Lately, inverse homogenization has been applied to the design of photonic isolaters by Christiansen et al. (2019). For more details on material design using inverse homogenization, we refer interested readers to the review papers by Cadman et al. (2013) and Osanov and Guest (2016) and the Ph.D. thesis by Andreassen (2015).

\section{Full-scale approaches}

As mentioned earlier, multi-scale structures can be designed using either multi-scale or mono-scale modelling. In this review, approaches based on multi-scale modelling (i.e., with the assumption of separation of length scales) are referred to as multi-scale approaches. Approaches that do not make this assumption, i.e., "mono-scale" approaches, optimize distribution of a homogeneous material. When the design domain is discretized by a finite-sized mesh, such "monoscale" approaches typically result in mono-scale structures. However, as the discretization of the design domain goes high, it can directly be used to achieve multi-scale structures, since theoretical stiffness optimal structures span multiple scales. By employing careful continuation techniques and sufficiently fine meshes, and in the absence of regularization for mesh independence such as control of minimum length, perimeter, or slope, multi-scale structures should appear naturally. However, the appearance of fine scale structures may also be stimulated by controlling the layout locally. In these approaches, analysis and optimization of structures are performed in the full resolution of the domain, and we thus refer to them as full-scale approaches.

For local control approaches, two strategies can be distinguished: pattern repetition and local volume constraints. In the former, the design domain is partitioned into a number of subdomains that are further refined. The layout in each subdomain is enforced to be the same as that in the others, leading to periodic patterns in the full domain. In a variation, the subdomains are grouped, and an identical layout is enforced in subdomains per group, resulting in, for instance, periodic patterns along one axis with gradation along another. The enforcement of an identical layout in subdomains can be achieved by creating a template that links all subdomains. The sensitivity of each point in the template is the sum of sensitivities of corresponding points in all linked subdomains. This strategy reduces the solution space from the full design domain to one subdomain, or a few for a gradation or partitioning, but the analysis is still performed on the full mesh.

Pattern repetition is pursued in full-scale approaches as well as multi-scale approaches. We discuss the former here, and will return to the latter in subsequent sections. Zhang and Sun (2006) presented a first and comprehensive study on creating periodic patterns using topology optimization. It was shown that topology design results were greatly influenced by both the number and aspect ratio of the subdomains. Similar results were later reproduced using evolutionary procedures (Huang and Xie 2008) and level sets (Liu et al. 2018b). Almeida et al. (2010) presented a density-based approach to create pattern repetition as well as structural symmetry. It is worth noting that the subdomains do not have to be of the same size. Repetitive pattern with a variation in size was demonstrated by Stromberg et al. (2011) and Wu et al. (2016a) for architectural design, by establishing a mapping between points in size-varying subdomains. Lately, the principle of pattern repetition has been used to design mechanisms (Wu et al. 2020). Designing periodic patterns using fullscale analysis involves intensive computation. Zhang and 
Sun (2006) proposed to reduce computation time through condensation - unfortunately, the details were missing in the original paper. Alexandersen and Lazarov (2015) showed an enormous reduction in computational cost by using a spectral coarse basis pre-conditioner that exploits the repeated patterns, i.e., for each repeated pattern the same spectral basis can be used.

The second strategy to stimulate fine scale structures is to apply local volume constraints (Wu et al. 2018). The idea is to impose an upper bound on the fraction of solid elements in the neighborhood of every point in the full design domain. This involves two important parameters: the radius of the neighborhood which affects the pore size, and the prescribed upper bound on the local volume fraction which controls the porosity. Since this creates a large number of constraints (equal to the number of elements), aggregation schemes such as the $p$-norm are often applied to aggregate the per-element constraints into a global one, facilitating the optimization process. It is found that local volume constraints yield bone-like porous structures that are aligned with principal stress directions (see Fig. 2a). These structures are robust with respect to load variations, local material failure, and buckling, at the price of some decrease in stiffness. A typical way to ensure robustness is to model uncertainties such as material failure and load variations in topology optimization, resulting in distributed structures (e.g., Jansen et al. (2014)). This demands multiple finite element analyses per optimization iteration. In this regard, applying local volume constraints is an efficient alternative for robust design. The concept of local volume constraints is similar to maximum length scale control, which was introduced by Guest (2009) and later studied in Lazarov and Wang (2017) and Carstensen and Guest (2018).

A number of further developments of the local volume constraints have been proposed. Wu et al. (2017) combined it with a coating approach (Clausen et al. 2015a) to simultaneously evolve the (macroscale) structure and the microstructures therein, referred to as shell-infill composites. Gradation in the porosity and pore size was demonstrated by Schmidt et al. (2019). Dou (2020) reformulated the constraints by a projection method, while Cang et al. (2019) made use of machine learning algorithms to predict optimized structures with local volume constraints. This was recently extended to incorporate multiple materials $(\mathrm{Li}$ et al. 2020). Furthermore, the local volume constraints have been applied in the context of other physics problems, e.g., heat conduction (Yan et al. 2018) and structural mechanics coupled with heat conduction (Das and Sutradhar 2020).

Figure 4 compares compliance-minimized structures optimized using a conventional mono-scale formulation under a total volume constraint (left), with pattern repetition (middle) and with local volume constraints (right). From this comparison it can be concluded that:

- Both pattern repetition and local volume constraints restrict the solution space, and the structure is expected to be less optimal than obtained from a formulation on the same resolution without these constraints. While more tests are not included here, it can be found that the restriction becomes more pronounced if the size of the repetitive pattern or the filter radius for defining local volume is reduced. Oppositely, when the pattern size or filter radius is larger than the size of the domain, the constraints have no influence on the solution; the local volume constraints degenerate to the conventional global volume constraint.

- Porous structures from local volume constraints exhibit continuous variations in orientation, while periodic patterns have a constant orientation. In this sense, local volume constraints are less restrictive than pattern repetition in constraining the optimization problem. Note that pattern repetition also implicitly controls the volume fraction in subdomains.

\section{Classification of multi-scale approaches}

Many works on multi-scale optimization repeat the theory to do numerical homogenization using finite elements, which
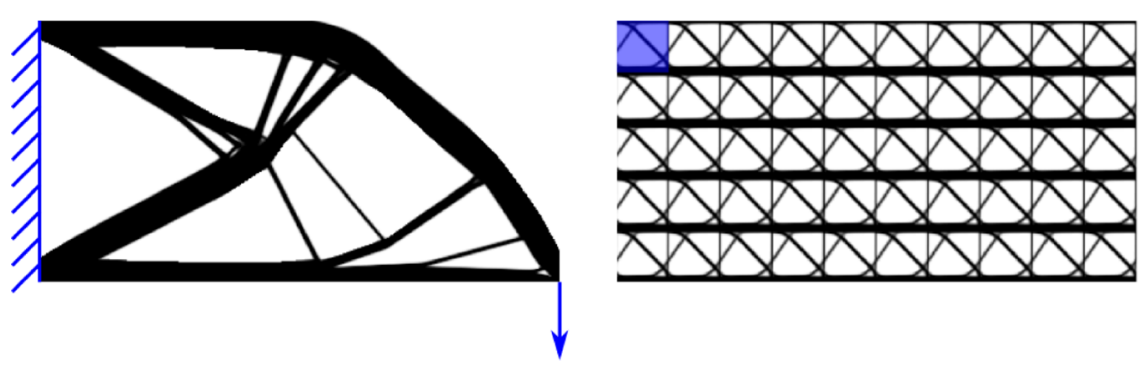

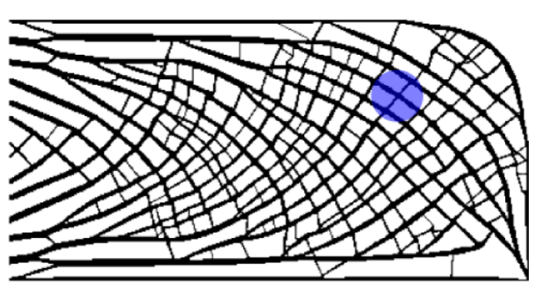

Fig. 4 Full-scale optimized structures using the same amount of material. The design space is a rectangular domain discretized by $400 \times 200$ square elements. The boundary condition is illustrated on the reference design (left). Middle: Optimized design with enforced pattern repetition. The size of the pattern is $40 \times 40$, indicated by a blue transparent square. Right: Optimized design with local volume constraints. The radius of the filter for calculating local volumes is 20 , indicated by a blue transparent disk. From left to right, the compliance values are $100.56,282.82$, and 152.28 , respectively 
was described in detail by Guedes and Kikuchi (1990). Based on this theory, Andreassen and Andreasen (2014) provided an open-source educational Matlab code to do numerical homogenization for plane problems, which has later been extended to 3D by Dong et al. (2019). However, more important is how to accurately define the multi-scale optimization problem. Therefore, we will now review some theoretical aspects about the problem formulation for compliance minimization problems for $n_{\text {load }}$ load cases. Using spatially varying microstructures, we can write the corresponding optimization problem as (e.g., Rodrigues et al. 2002),

$$
\max _{\rho} \max _{\rho} \min _{\rho \mathrm{d} \Omega \leq V_{\max }}\left\{\sum_{k=1}^{n_{\text {load }}} w^{k} \Pi_{\text {tot }}^{k}\right\} .
$$

Here, $\rho$ is the macroscale variable describing the porosity of the varying microstructures, which is subject to an upper bound on the available material $V_{\max } . \boldsymbol{E}^{H}$ describes the homogenized elasticity tensor of the microstructures, which has to be in the physically admissible set of elasticity tensors $\boldsymbol{E}_{a d}$. The inner problem describes the minimization of potential energy $\Pi_{t o t}^{k}$, i.e., the equilibrium constraint that has to hold for each load case $k$, where we solve for displacement field $\boldsymbol{u}^{k}$ in the space of kinematically admissible displacement fields $\boldsymbol{U}$. Finally, $w^{k}$ is a weighting factor to scale the energy for each load case. The compliance $\mathcal{J}$ can be calculated as,

$\mathcal{J}=-2\left\{\sum_{k=1}^{n_{\text {load }}} w^{k} \Pi_{\text {tot }}^{k}\right\}$,

hence, maximizing the total potential energy is equivalent to minimizing the compliance.

Optimization problem (1) can be solved in different ways, e.g., as one system of equations using the simultaneous analysis and design (SAND) approach (Jog and Haber 1996), or using a sequence of separable convex approximations using a so-called nested analysis and design (NAND) approach (Fleury 1993). The latter is the de facto standard for topology optimization problems. However, solving problem (1) would, depending on the way the material of the microstructures is modeled, result in a large KKT matrix for the maximization with respect to $\boldsymbol{E}^{H}$. To circumvent this, we can interchange the maximization with respect to $\boldsymbol{E}^{H}$ and the minimization with respect to $\boldsymbol{u}$,

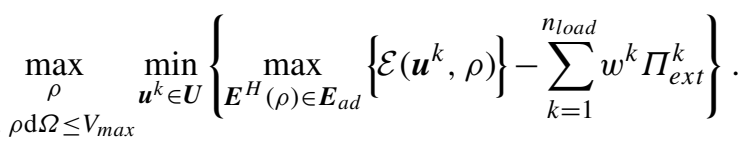

Here, $\Pi_{\text {ext }}$ is the potential energy of the applied loads for the $k$-th load case, and the point-wise optimal strain energy $\mathcal{E}$ can be written as,

$\mathcal{E}=\frac{1}{2} \sum_{k=1}^{n_{\text {load }}} w^{k}\left(\int_{\Omega} \boldsymbol{\varepsilon}\left(\boldsymbol{u}^{k}\right): \boldsymbol{E}^{H}(\boldsymbol{\rho}): \boldsymbol{\varepsilon}\left(\boldsymbol{u}^{k}\right) \mathrm{d} \Omega\right)$.
Hence, we now have an inner point-wise microstructure optimization problem. As proven by Lipton (1994b), problem (3) is equivalent to problem (1) if a microstructure parameterization is used such that the strain energy is concave with respect to $\boldsymbol{E}^{H}$. This concavity is a property of optimal rank- $N$ microstructures. Hence, if suboptimal microstructures are used, the optimality of the solution to problem (3) cannot be guaranteed. Nevertheless, problem (3) results in an optimization problem that is independent of the material description and thus forms the basis for the vast majority of multi-scale topology optimization approaches. The most common way to solve the optimization problem (3) is to use a hierarchical solution procedure (see, e.g., Bendsøe and Sigmund (2004) for a detailed description), which can be summarized in the flowchart below.

1. Initialize data: Geometry, mesh, boundary conditions, density $\rho$, microstructure description $\boldsymbol{E}^{H}$, etc.

2. Analysis: Solve for macroscale displacements $\boldsymbol{u}^{k}$.

3. Microstructure optimization: Solve point-wise for $\boldsymbol{E}^{H}$ by maximizing $\mathcal{E}$ for given strains and $\rho$.

4. Density update: Update the outer maximization problem, subject to volume constraint.

5. Check convergence: Check if the optimization problem is converged. If not, repeat steps $2-5$.

It is not necessary to solve the problem exactly like this. For example, one can combine the global density update and all local problems in one large optimization step (see, e.g., Bendsøe and Kikuchi (1988)). However, for problems with a large number of design variables, the separation in local and global problems allows for reducing the computational time through the use of parallel computations (Coelho et al. 2008).

An alternative to the hierarchical formulation (problem (1)) is to assign two sets of design variables, i.e., on both macro- and microscales, and concurrently optimize these two sets. This concurrent formulation, known as PAMP (Porous Anisotropic Material with Penalization), was proposed by Liu et al. (2008) to design microstructures and their macroscopic layout.

Problem (3) and the abovementioned flowchart will be used to categorize multi-scale approaches later on. Although the equation concerns compliance minimization in the elasticity setting, similar looking equations and flowcharts can be made for different types of physics including additional constraints. Each of these multi-scale problems can be divided into a sequence of three related optimization problems, i.e., a state problem; a global parameter distribution problem; and one or many local microstructure optimization problems. The key point in classifying the different multi-scale topology optimization 
approaches is to determine what kinds of restrictions are applied to the solution space of each of the sub-problems. For now, we assume that at least the state problem is solved up to full accuracy in each of the multi-scale problems, although this may not apply to deep learning or iterative solution approaches. Hence, we classify the different approaches by (1) the restrictions that are applied to the density distribution and (2) the restrictions that are applied to the admissible set of properties $\boldsymbol{E}_{a d}$ that can be achieved by the parameterized microstructure.

It should be mentioned that even in the elasticity setting it is not for all cases exactly known which constitutive tensors are realizable (Milton et al. 2017). For example, microstructures that reach minimum shear modulus at the Walpole point have never been realized (Sigmund 2000). However, as discussed above, rank- $N$ laminates cover the full space of optimal designs for compliance minimization problems. In other words, if one uses rank- $N$ laminates for the microstructure description one can solve problem (3) to the true optimum. It is known that for a single load case in a 2D setting, rank-2 laminates are sufficient to describe the optimal solution, while for multiple load cases a rank-3 laminate is required (Avellaneda 1987). Hence, if one would only use a rank-2 parameterization for a multiple load case problem, the optimality could be severely reduced. Since the vast majority of works on multi-scale topology optimization use (strongly) restricted sets $\boldsymbol{E}_{a d}$ (for computational, manufacturing or other reasons), we categorize approaches based on the following restrictions, starting with the least restricted category:

I Optimal set of elasticity tensors: $\boldsymbol{E}_{a d}$ is represented by a geometry parameterization that allows the local problem to be solved to optimality. This is for elasticity and conduction problems the set of rank- $N$ laminates (Allaire 2002). However, note that, for many other types of problems, the optimal set of constitutive tensors is not exactly known yet.

II Unrestricted unit-cell design: $\boldsymbol{E}_{a d}$ contains the set of unit cells that can be obtained using inverse homogenization, without restrictions on the material distribution, shape, connectivity, or orientation of the unit cell. This means that if a fine enough discretization is used, the microstructures should converge to what is theoretically possible. However, in practice, limited mesh resolution will cause suboptimal microstructures.

III Restricted unit-cell design: $\boldsymbol{E}_{a d}$ contains the set of unit cells that can be obtained using inverse homogenization with restrictions on material distribution, unit-cell shape, connectivity, or orientation. For example, this can be a square unit cell or a design with pre-defined solid elements, both resulting in severely restricted design freedom.
IV Parameterized unit cell with multiple parameters: $\boldsymbol{E}_{a d}$ contains a set of pre-computed parameterized unit cells such that the microstructure is parameterized using more than 1 parameter. For example, this can be the rectangular hole microstructure by Bendsøe and Kikuchi (1988). Due to the rotational freedom, the rectangular hole cell actually performs almost as well as rank-2 microstructures (Bendsøe and Sigmund 1999), if properly de-homogenized.

V Parameterized unit cell based on density: $\boldsymbol{E}_{a d}$ contains a single constitutive tensor $\boldsymbol{E}^{H}$ for a given microstructure density $\rho$. This is the most restrictive case since it does not involve a local optimization problem. Isotropic microstructures satisfying the HashinShtrikman bounds (Hashin and Shtrikman 1963) fall in this category since the isotropic elasticity tensor follows only from the density. Likewise, the SIMP approach with a penalty parameter $p$ that satisfies the Hashin-Shtrikman bounds (Bendsøe and Sigmund 1999) falls into this category.

The difference between category III where the microstructure topology is optimized during the optimization process and category IV is that for category IV a database of pre-computed microstructural properties is generated. Hence, category IV will require a heavy precomputation step, but afterwards the optimization problem can be solved more efficiently.

There are many works that consider a set of elasticity tensors $\boldsymbol{E}_{a d}$ outside of the bounds on what is theoretically possible. An example of this is the free material optimization (FMO) approach (Bendsøe et al. 1994), which has also been used in two-scale structural optimization (Schury et al. 2012; $\mathrm{Hu}$ et al. 2020). In this method, the entries of $\boldsymbol{E}^{H}$ are the design variables, and using either a constraint on a norm or on eigenvalues of $\boldsymbol{E}^{H}$ one can try to relate the tensor to a density distribution. Nevertheless, there is no direct relation between the bounds used on the tensor and the Hashin-Shtrikman bounds; and therefore, we will not discuss the FMO method further in this review. Another good example of violation of the bounds is the variable thickness sheet problem (Rossow and Taylor 1973), i.e., the SIMP method with $p=1$. In 2 dimensions, we can justify these designs by interpreting density as a thickness in the outof-plane direction. Hence, it should therefore be seen as a sizing problem. However, the model becomes invalid if used to pre-compute a global density distribution for a subsequent porous microstructure realization. One cannot make an in-plane realization of these variable thickness designs and therefore works that use SIMP with $p=1$ for any porous or 3D design problem should be avoided.

Finally, we can categorize the outer problem into three different categories. 
A Unrestricted density: There are no restrictions on the density, i.e., $\rho \in[0,1]$.

B Restricted density: Only a few values of $\rho$ are allowed. For example, this applies to the SIMP method (possibly combined with a projection method (Guest et al. 2004) to reduce elements with intermediate densities), and also the PAMP approach (Liu et al. 2008) falls into this category. This also applies to interface bounded approaches (Clausen et al. 2015a; Groen et al. 2019; Luo et al. 2019) with a fixed infill density and a solid outer shell.

C Fixed density: The density field is fixed, i.e., there is no outer optimization problem. This is, for example, a uniform density field or a density distribution based on some prior optimization problem. Note that a fixed density does not necessarily restrict the unit cell to be periodic, though this is the case in Cherkaev et al. (1998) and Fujii et al. (2001). A fixed density permits unit cell adaptation, e.g., rotation (Wu et al. 2021).

In total, we can thus identify $5 \times 3$ different categories of multi-scale topology optimization problems, which are summarized in Table 1. Here, we also list a fundamental paper for each category (if available).

\section{Multi-scale approaches}

This section is organized in accordance with the above classification based on restrictions on unit cells. We start with the least restricted category-the optimal set of constitutive properties (I), and then proceed directly to the most restricted one where unit cells are parameterized by a single parameter, i.e., density (V). This is followed by categories with increasing flexibility in the unit cell parameterization (II, III, IV). This order is found to align with the chronological order of the first article in each category (see Table 1, column A).

\subsection{Optimal set of constitutive properties}

In the context of elasticity, the minimum number of sequential layers that is required to parameterize the optimal solution using rank- $N$ microstructures depends on both the number of load cases and the dimension of the problem (Avellaneda 1987; Francfort and Murat 1986):

- Rank-2 laminates with orthogonal layers, for plane problems subject to a single load case

- Rank-3 laminates, for plane problems subject to multiple load cases

- Rank-3 laminates with orthogonal layers, for problems in 3D subject to a single load case

- Rank-6 laminates for problems in 3D subject to multiple load cases

Hence, at most, 7 different length scales are needed to describe an optimal material parameterization in $3 \mathrm{D}$, i.e., six length scales for the microstructure and one for the macroscopic parameterization. The elasticity tensors of these multi-scale laminates can be derived analytically, allowing the local microstructure optimization problems to be solved in a very efficient manner. For example, for a rank-2 laminate shown in Fig. 5 (right) the first layering is constructed on the infinitesimal length scale $x / \epsilon^{2}$ shown in Fig. 5 (left). This layering can be described by the relative layer width $\mu_{1}$ that describes the ratio of the isotropic stiff material $(+)$ compared to the weak void material (-) and the interface normal $n^{1}$ and tangent $t^{1}$. Subsequently, the rank-2 laminate is constructed using a second layering on length scale $x / \epsilon$ using relative layer width $\mu_{2}$ that describes the ratio of the stiff material to the first layering. By ensuring orthogonal interface vectors, the elasticity tensor is thus defined by only three parameters, $\mu_{1}, \mu_{2}$, and $\theta_{2}$, besides the material properties of the stiff (+) and weak (-) phase respectively.

The first work in which topology optimization using rank-2 laminates was considered is the work by Bendsøe (1989). Subsequently, Allaire and Kohn (1993) proved that the optimal design for plane problem subject to a single load case can be described purely by the stress distribution in the macroscopic domain. Multiple load case problems, where the design is parameterized using optimal rank-3 laminates, have been considered for both plane problems (Allaire et al. 1996; Cherkaev et al. 1998) and plate/shell problems (Díaz et al. 1995; Hammer et al. 1997; Krog and Olhoff 1999). By choosing different plate layouts, e.g., using a fixed core thickness, Soto and Díaz (1993) and Krog and Olhoff (1999)

Table 1 Categories of multi-scale topology optimization problems and representative papers

\begin{tabular}{|c|c|c|c|}
\hline & A: Unrestricted density & B: Restricted density & C: Fixed density \\
\hline I: Optimal set of elasticity tensors & Rank-2 (Bendsøe 1989) & Soto and Díaz (1993) & Cherkaev et al. (1998) \\
\hline II: Unrestricted unit-cell design & Barbarosie and Toader (2014) & - & - \\
\hline III: Restricted unit-cell design & Rodrigues et al. (2002) & Liu et al. (2008) & Fujii et al. (2001) \\
\hline IV: Parameterized unit cell with multiple parameters & Bendsøe and Kikuchi (1988) & Groen et al. (2019) & Wu et al. (2021) \\
\hline V: Parameterized unit cell based on density & SIMP (Bendsøe 1989) & Guest et al. (2004) & Finite element analysis \\
\hline
\end{tabular}


Fig. 5 Visualization of how a rank-2 microstructure is constructed from a stiff isotropic material (+) and a weak/compliant isotropic material (-). Please note the different length scales, on the left $x / \epsilon^{2}$ and on the right $x / \epsilon$, with the characteristic unit-cell length $\epsilon \rightarrow 0$
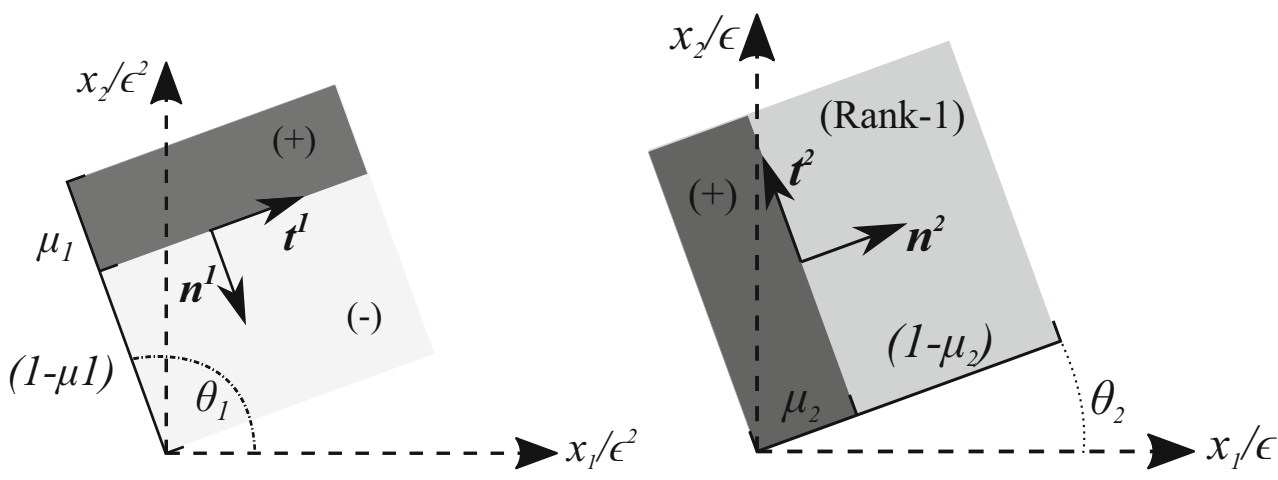

showed the effect of using several density restrictions on the performance. The effect of restricting the amount of unique microstructures in the domain has been investigated by Cherkaev et al. (1998), who demonstrated that the performance can be at least twice as bad if only 1 or a few unique micostructures are used in the design domain.

The method has been applied to 3D topology optimization problems, where orthogonal rank-3 laminates are used for single load case problems (Cherkaev and Palais 1996; Allaire et al. 1997; Díaz and Lipton 1997; Olhoff et al. 1998; Czarnecki and Lewiński 2006). Multiple load cases are considered by Díaz and Lipton (2000). A nice feature of rank- $N$ laminates is that they provide a lower bound on the theoretical compliance that can be reached. It is thus recommended to include this comparison when presenting a multi-scale approach. To help doing so, we supply a code for the optimization of rank-2 microstructures, which is discussed in Appendix.

A downside of structures optimized using rank- $N$ laminates is that they consist of several length scales. This poses two challenges. Firstly, although technology is rapidly developing and lab work has demonstrated multiscale manufacturing capability (e.g., Zheng et al. (2016)), a majority of manufacturing techniques do not yet support precise production of structures spanning multiple length scales. Hence, single scale interpretation of multi-scale rank- $N$ laminates is required. Träff et al. (2019) have shown that the multi-scale rank- $N$ microstructures can be approximated on a single scale with only a small loss in performance. Secondly, it is necessary to compile globally consistent structures from locally defined rank- $N$ laminates (or their simplified versions), i.e., to de-homogenize the results. This problem was first addressed by Pantz and Trabelsi (2008), who used an implicit geometry description to interpret the optimized multi-scale designs on a single length scale with little loss in performance. More details on de-homogenization will be presented later in Section 5.3.1.

Another difficulty with the interpretation of rank- $N$ microstructures is that, for multiple load cases, there exist an infinite number of different microstructure parameterizations that can reach the same elasticity tensor. As an example, consider the planar unit-cell optimization problem using four independent load cases shown in Fig. 6 (top). If we optimize for minimum complementary energy and find the corresponding rank-3 laminates using the method of Lipton (1994a), we obtain the four distinct microstructure realizations shown in Fig. 6 (bottom). These microstructures and an infinite number of combinations thereof can
Fig. 6 Top: Illustration of the four stress cases and their respective weights. Bottom: Visualizations of optimal rank-3 laminates with indicated hierarchy, optimized for the 4 stress cases using $\rho=0.5$ (Groen 2019)
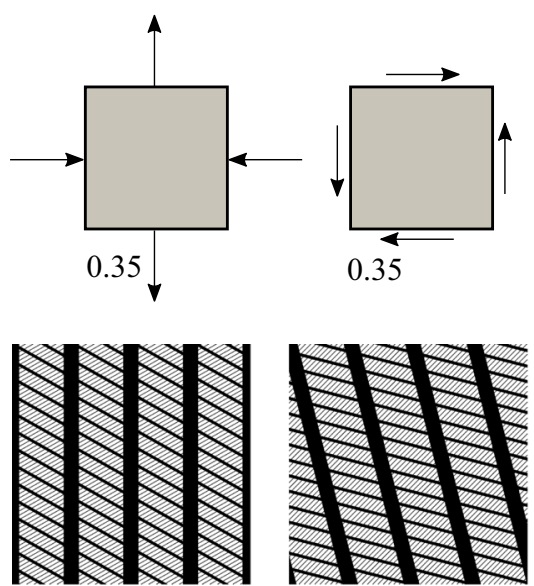

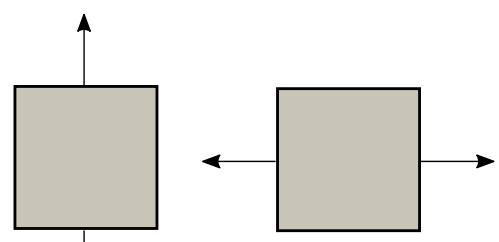

0.15 
Fig. 7 Three types of 3D unit cells, from left to right, truss- or beam-like lattice structure, triply periodic minimal surface (TPMS), and topology optimized microstructure (Reprinted from Andreassen et al. 2014 with permission from Elsevier)

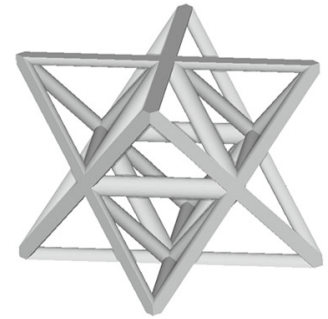

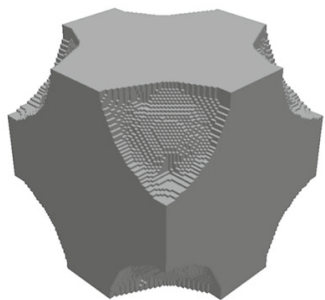

achieve the same minimum complementary energy, which is both an advantage and a difficulty when finding practical interpretations of rank- $N$ designs.

Finally, it should be mentioned again that for many problems beyond elasticity and heat conduction, a full parameterization of the optimal set of constitutive properties is yet to be found. Finding these bounds on the properties and geometries that reach these bounds is a research field on its own. The interested reader is referred to the books on the theory of composites by Milton $(2002,2016)$.

\subsection{Parameterized unit cell based on density}

Predefined cellular structures such as those shown in Fig. 7 have been used to efficiently design multiscale components. Common in many of these multi-scale approaches is the use of homogenization to evaluate the effective material properties of predefined unit cells. Since the cells are predefined, homogenization can be performed off-line prior to optimization. This allows to generate structures with fine geometric details at a runtime computational complexity comparable to mono-scale approaches (Section 2.2). This is attractive, as evidenced by the large number of publications and industrial examples falling into this category. Predefined unit cells reduce the solution space of the microstructures to a dimension of 1 (or a few, see Section 5.3). This distinguishes this approach from hierarchical or concurrent approaches (Sections 5.4 and 5.5), where microstructures are represented by multivariable density fields. This distinction is meaningful for implementation. Since the solution space is reduced, it is convenient to construct a differentiable function that maps the reduced parameters to homogenized properties, and use this function in macroscale optimization.

A typical application of these approaches is to design 3D printed components with uniform infill patterns. From the optimization perspective, this requires no or little adaption of mono-scale optimization approaches. The repeating unit cell can be interpreted as a material, the distribution of which is then optimized by mono-scale approaches. Note that the unit cells interpreted as a material can be isotropic or anisotropic. An example of the former is a 2D beam-like lattice structure constructed from a regular triangular tiling (Clausen et al. 2016). In contrast, the 2D lattice structure from a regular square tiling is anisotropic. Most mono-scale optimization approaches in their standard form deal with isotropic materials. To handle anisotropic materials, the stiffness matrices in finite element analysis must be adapted to reflect the homogenized elasticity tensor of the anisotropic material. Also note that if not optimally oriented along local principal stress directions, anisotropic microstructures may result in highly suboptimal results.

There has been a growing interest in designing components with graded cellular structures. Here, the input to optimization extends from a single unit cell to a set of cells with gradation in porosity and effective material properties (e.g., elasticity tensor). This set can thus be referred to as functionally graded cells. They are typically parameterized by the fraction of solid material within each cell $(\rho)$, naturally serving as the design variable in optimization. The homogenized elasticity tensor $\left(\boldsymbol{E}^{H}\right)$ is a function of $\rho, \boldsymbol{E}^{H}(\rho)$, which can be constructed by interpolating elasticity tensors of sample cells with equally spaced material fractions. $\boldsymbol{E}^{H}(\rho)$ thus depends on the specific functionally graded cells, and in general it deviates from the powerlaw relation. This function for $\mathrm{X}$-shaped lattice structures with varying thicknesses is visualized in Fig. 8a, where the power-law curves with $p=1$ (i.e., unpenalized), 2 and 3 are also plotted. For accurately representing the corresponding mechanical properties, the cell-specific $\boldsymbol{E}^{H}(\rho)$ shall be used in lieu of the generic power-law in density-based topology optimization. The cell-specific $\boldsymbol{E}^{H}(\rho)$ is commonly constructed using numerical homogenization. Wu et al. (2019) proposed an alternative surrogate model for mapping the effective properties of density parameterized unit cells. It used static condensation to reduce the degrees of freedom of unit cells, followed by proper orthogonal decomposition and diffuse approximation for mapping the density to unit cell stiffness matrix.

Cell-specific material interpolation models have been used in the optimization for components consisting of gyroid-based cellular structures (Li et al. 2018a) and beamlike lattice structures (Wang et al. 2018; Watts et al. 2019), as well as topology optimized microstructures (Garner et al. 2019). Graded cellular structures can also be obtained by post-processing an unmodified SIMP-based mono-scale topology optimization approach, e.g., by replacing the intermediate densities by unit cells of the corresponding 


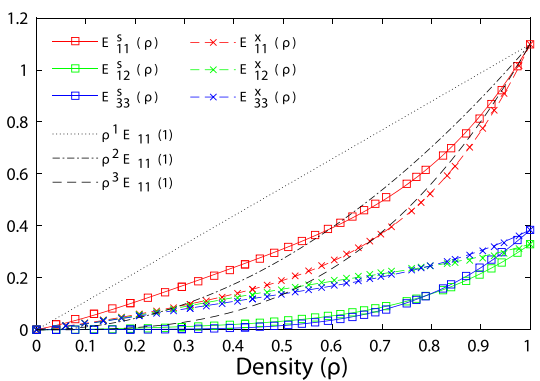

a) Interpolation functions

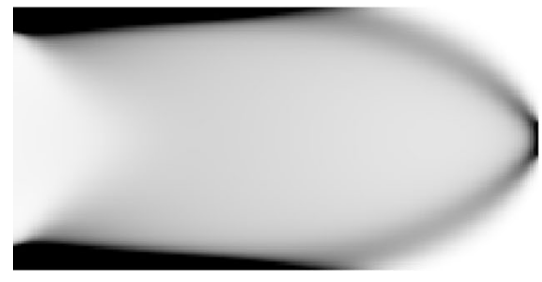

d) SIMP, $p=1 . c=95.92$

$c^{s}=1017.03, c^{x}=276.11$

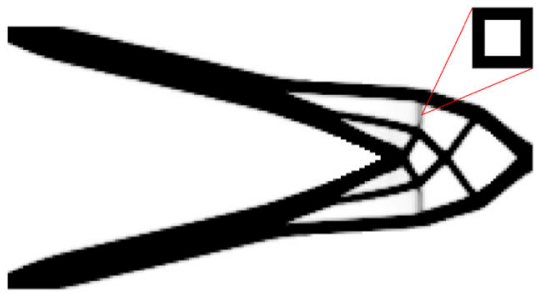

b) Square-shaped cell. $c^{5}=139.41$

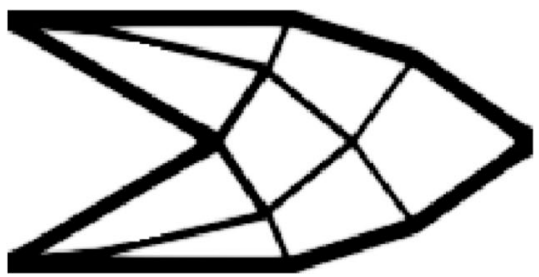

e) SIMP, $p=3 . c=118.00$

$c^{s}=118.31, c^{x}=115.29$

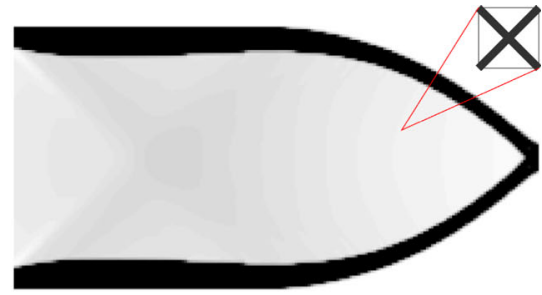

c) X-shaped cell. $c^{\mathrm{X}}=125.24$

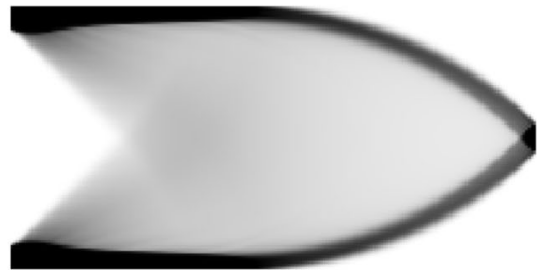

f) Rank- 2 material. $c=104.65$
Fig. 8 Comparison of different interpolations for topology optimization of a cantilever beam using a volume fraction of $25 \%$. a Interpolation functions for square-shaped $\left(E^{s}\right)$ and X-shaped $\left(E^{x}\right)$ lattice cells with varying member thicknesses. The samples of square- and $\mathrm{X}$-shaped cells are marked by $\square$ and $\times$, respectively. b, c Optimized density distributions using square- and X-shaped cells with cell-specific interpolation functions, respectively. d, e Optimized

material fractions (Brackett et al. 2011; Panesar et al. 2018). Figure 8 compares interpolation schemes for two different unit cells (c.f., (b) and (c)) with varying member thicknesses. The comparison is performed on the 2D cantilever problem, thoroughly studied by Sigmund et al. (2016). The domain is fixed at its left edge and loaded with a unit vertical load distributed over the central $10 \%$ of the right edge. Young's moduli for the solid and void material are $E_{0}=1$ and $E_{\min }=0.001$, respectively ${ }^{1}$. Poisson's ratio is $v_{0}=0.3$ for both materials ${ }^{2}$. The compliances of the designs using the cell-specific interpolation functions (b and c) are much smaller than the design using generic (unphysical) power-law with $p=1$ (d) and post-processed by interpreting gray elements with square- or X-shaped cells, demonstrating the significance of using an accurate material interpolation model in optimization. It simply does not make mechanical sense to use a non-physical macroscale model like SIMP with $p=1$ to control the macroscopic density in multi-scale approaches. In (d), the post-process of replacing gray elements by square-shaped cells leads to a large compliance of $c^{s}=1017.03$, since low-density square-shaped cells are weak in shear. The designs using cell-specific interpolations $(b$ and $c$ ) have

\footnotetext{
${ }^{1}$ For rank-2 material, continuation can be applied to allow for a smaller value of $E_{\min }$; see Appendix.

${ }^{2} v_{0}=0.0$ was used in Sigmund et al. (2016)
}

density distributions using a SIMP interpolation, with $p=1$ and 3, respectively. In $\mathbf{d}$ and $\mathbf{e}, c$ indicates the compliance estimated by the generic (unphysical) power-law, while $c^{s}$ and $c^{x}$ refer to the compliance re-evaluated with cell-specific elasticity tensors. SIMP with $p=1$ d significantly overestimates the stiffness of lattice cells. $\mathbf{f}$ A reference design by using rank- 2 material (obtained with code in Appendix)

higher compliance values than design (e) using the power law with $p=3$ ( $p$ starts from 1 and is increased by 0.2 every 50 iterations). This suggests the optimization using cell-specific interpolations gets stuck in local minima. The number of iterations for obtaining both (b) and (c) is 400 . Design (b) has a density distribution close to black and white, while design (c) has large gray regions. X-Shaped cells have large shear moduli, making it economical to place gray elements in the middle of the beam which is predominately under shear stress. Rank- 2 material achieves optimal stiffness (f) due to the alignment of material anisotropy with principal stress directions, whereas the fixed orientation of the square- and X-shaped lattice cells restricts the adaptation of material anisotropy.

The curves for $E_{i j}^{H}(\rho)$ for the square- and X-shaped cells are lower than the theoretical limit according to the Hashin-Shtrikman bounds (Hashin and Shtrikman 1963). This means a suboptimal use of material using cellular structures with a simple geometry. To improve the properties, Zhou and $\mathrm{Li}$ (2008) applied inverse homogenization (see Section 2.3) to design a series of unit cells with varying material fractions. Figure 9 (top) shows unit cells independently optimized for an increasing material fraction, using the code provided by Xia and Breitkopf (2015). These optimized cells have distinct topology, owing to the large solution space in topology optimization. It is also noticeable that neighboring cells, 

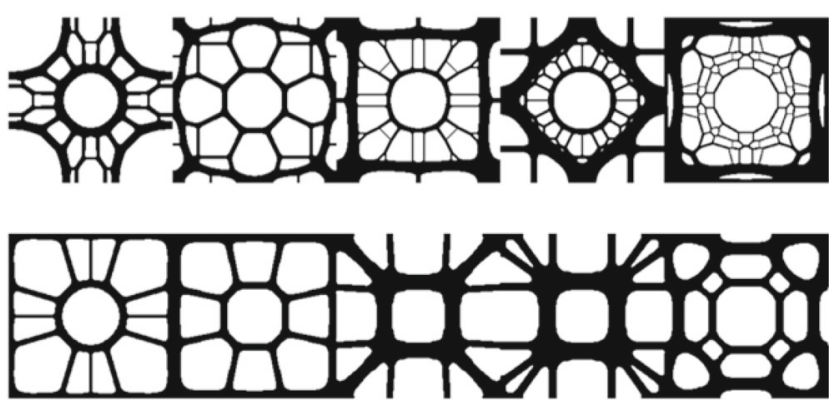

Fig. 9 Top: Illustration of poorly connected microstructures. The unit cells are individually optimized for maximum bulk modulus under linearly varying volume fractions from 30 to $50 \%$, from left to right. Bottom: Optimized connectivity, achieved by maximizing the bulk modulus of extended domains covering adjacent cells (Garner et al. 2019). Reprinted from Garner et al. (2019) with permission from Elsevier

despite being close in terms of material fractions, may have poor connectivity across the interface (see Fig. 9 (top), between the first and second and between the third and fourth cells). The connectivity issue is also recognizable in hierarchical approaches (Rodrigues et al. 2002), to be discussed in Section 5.4. Note that this is less of a problem in cellular structures parameterized by thickness since the topology there is constant, as long as the thickness does not vanish to 0 .

Zhou and $\mathrm{Li}$ (2008) proposed three methods to address the connectivity issue between topology optimized microstructures: kinematic connectivity constraint, pseudo load, and unified formulation with non-linear diffusion. In the first two, unit cells are optimized individually, while solid non-design regions or pseudo loads are prescribed along the domain boundary to stimulate connectivity. In the last, unit cells are optimized altogether, while a nonlinear diffusion term defined on the domain covering all cells is integrated in the objective function to penalize disconnection and suppress checkerboard patterns. Radman (2013a, b) improved the computational efficiency of the unified formulation by successively optimizing new unit cells, while considering connection to already optimized cells. Du et al. (2018) proposed a physics-independent connectivity index, which measures the amount of overlap in adjacent cells across the shared interface. Garner et al. (2019) proposed to optimize the connectivity, quantified by the physical properties of interest (e.g., bulk modulus) of an extended domain that covers adjacent cells. Poor connectivity between adjacent cells leads to an inferior bulk modulus for the extended domain as a compound cell, and thus is effectively suppressed in optimization. Figure 9 (bottom) shows results from this compound formulation. Mapping these compatibility optimized cells to a compliance-minimized macroscale density distribution, and evaluating structural compliance using both full-scale and homogenization-based analyses, it was found that the discrepancy is small (a relative error of 2\%). This small error is also attributed to the limited and slowly varying nature of the microstructure which satisfies basic assumptions of the homogenization-based multi-scale modelling. This method has been demonstrated for optimizing up to 100 varying unit cells in 2D. Optimizing a large number of unit cells in 3D can be computationally expensive. Cramer et al. (2016) proposed geometric interpolation to obtain transitioning microstructures between individually optimized unit cells. This geometric approach works for microstructures of similar topology.

Cellular structures, both assemblies of geometric primitives and topology optimized microstructures, are typically defined within a square or cube domain. Such a fixed design domain may limit the achievable properties (c.f., Träff et al. (2019)). These cellular structures may be square symmetric (2D) or cubic symmetric (3D) but not (necessarily) isotropic. If orthotropic directions are oriented along regular finite element grid directions and not along principal stress directions as recommended by Pedersen (1989), inferior stiffness may be the result.

\subsection{Parameterized unit cell with multiple parameters}

Functionally graded cells can be obtained by uniformly varying the thickness of the geometric primitives in the cell domain, e.g., the square- and X-shaped 2D lattices in the previous subsection. Consequently, each element in the macroscale optimization is assigned a single design variable, the material volume fraction, $\rho(d)$, where $d$ is the thickness. To enlarge the solution space, the parameterization of unit cells can be extended. The unit cell in Fig. 10 (middle) has two superimposed geometric patterns, i.e., an $\mathrm{X}$ shape and a plus shape, each with an independent thickness, leading to more variations in the attainable elasticity tensor, $\boldsymbol{E}^{H}(d, t)$. The number of design variables per element can be further increased, e.g., by assigning an individual thickness per geometric primitive, indicated by different colors in Fig. 10 (right). Graded lattice structures in 2D and 3D optimized with multiple design variables per macro element have been demonstrated by Wang $(2018,2020)$. Imediegwu et al. (2019) used a lattice cell with seven independent parameters for 3D optimization. As the number of independent parameters
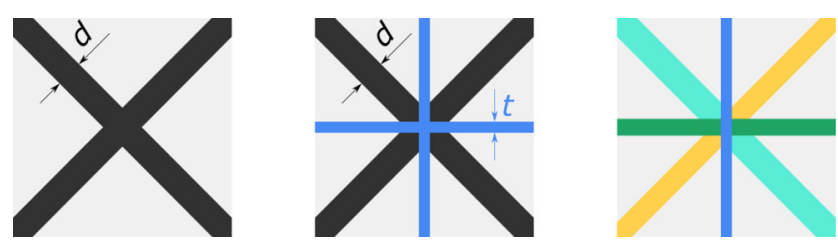

Fig. 10 Illustration of unit cells with 1,2 , and 4 independent parameters, from left to right 


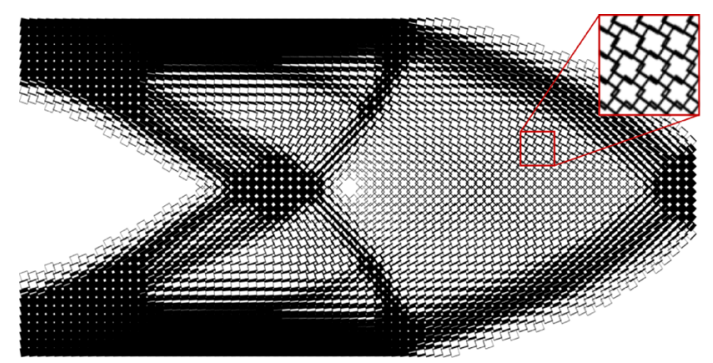

Fig. 11 Single-scale interpretations of a multi-scale design for the Michell cantilever using a volume fraction of 0.4 and the microstructures by Bendsøe and Kikuchi (1988). Left: Naive interpretation, right:

for describing the unit cell increases, a large number of numerical homogenization operations is required. White et al. (2019) proposed a neural network surrogate model to provide the mapping from parameters to resulting metamaterial properties. Zhu et al. (2017) pre-computed the space of attainable Young's modulus and Poisson's ratio for certain types of microstructures, and used this space as a constraint for macroscale topology optimization with microstructures. Zhu et al. (2019) presented an approach for concurrent optimization of the microscale material distribution and a macroscale mapping function which transforms periodic microstructures into graded ones. This was extended and demonstrated for 3D problems (Xue et al. 2020).

As the number of parameters increases, the potential to generate a variety in the anisotropic directions of the microstructures increases, and this, to some extent, allows an adaption of microstructural anisotropy to the local stress directions during optimization. To ultimately align the anisotropy of microstructures to stress directions, it is critical to release the rotational freedom in the unit cell parameterization, as pioneered by Bendsøe and Kikuchi (1988).

\subsubsection{De-homogenization}

A benefit of the approaches using parameterized unit cells with multiple parameters including rotation is that one can get optimized designs that perform very close to the theoretical limit. For example, let us consider a classical optimization example: the Michell cantilever clamped on the left with a unit load on $20 \%$ of the right boundary. Using the square microstructure with a rectangular hole by Bendsøe and Kikuchi (1988) shown in Fig. 3, and parameterizing the design domain by $80 \times 40$ bi-linear elements, we can obtain a compliance value of 58.35 for an upper bound on the material volume fraction of 0.4 . This is very close to the value of 56.73 obtained using optimal rank-2 microstructures reported in Sigmund et al. (2016). Although the microstructures are on a single length scale, it is still difficult to interpret the spatially varying multi-scale

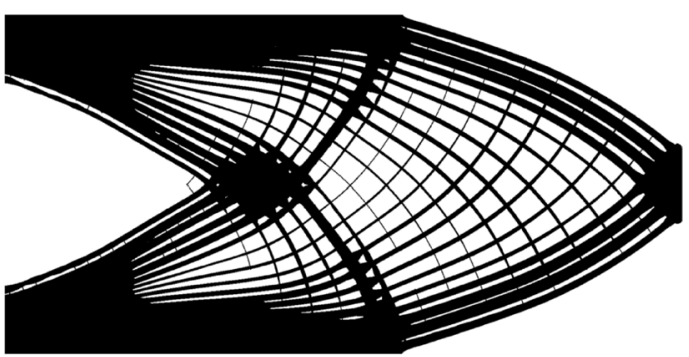

de-homogenized design using the approach presented in Groen and Sigmund (2018). Reprinted from Groen et al. (2019) with permission from John Wiley \& Sons

design as a well-connected manufacturable design. A naive approach would be to enlarge each microstructure to the size of a bi-linear element and apply the appropriate orientation. As can be seen in Fig. 11 (left), this results in a disconnected design. The process of constructing connected and physically realizable designs from homogenizationbased optimization is referred to as de-homogenization-a term coined by G. Allaire and colleagues.

A very promising post-processing method to obtain wellconnected mono-scale designs from a spatially varying multi-scale design is the de-homogenization method introduced by Pantz and Trabelsi (2008). Using this method, an implicit geometry description is created to represent/enlarge the multi-scale design to a fine but realizable single length scale. In recent years, the interest in this approach has renewed, resulting in simplifications and improvements of the approach (Groen and Sigmund 2018; Allaire et al. 2018). The approach of Groen and Sigmund (2018), applied to the Michell cantilever, resulted in the high-resolution mono-scale design $(1600 \times 800$ elements $)$ shown in Fig. 11 (right). A minimum feature size is applied and resulting compliance is 59.55. The core idea of the approach is to create a set of smooth mapping functions $\phi_{i}(\boldsymbol{x})$ that convert from the global frame of reference $\boldsymbol{x}$ to the microstructure frame of reference $\boldsymbol{y}$. In other words, we create a conformallike map in a similar fashion as texture mapping (Lévy et al. 2002). The most important requirement is that the spatially varying microstructure orientation is smooth throughout the domain such that smooth mapping functions $\phi_{i}(\boldsymbol{x})$ can be generated. A visual overview of how the approach works is given in Fig. 12. Consider a coated domain (Fig. 12a) with a porous interior using the microstructure in Fig. 3 with $\mu_{1}=$ $\mu_{2}=0.1$, the corresponding microstructure orientation $\theta$ is shown in Fig. 12b. One of the two mapping functions $\phi_{1}(\boldsymbol{x})$ is shown in Fig. 12c and the corresponding dehomogenized design is seen in Fig. 12d. A main advantage of this approach is that since it uses an implicit geometry description, the design can be evaluated at an infinitely fine resolution. Furthermore, the periodicity (i.e., the amount of microstructures) can be explicitly controlled, even to have 

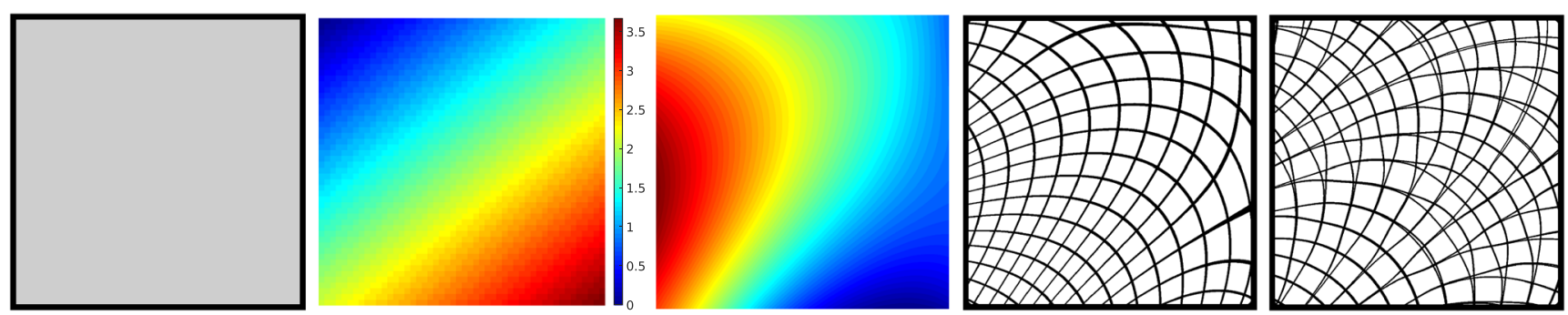

Fig. 12 Example of the de-homogenization procedure. From left to right, a coated domain, $\mathbf{b}$ microstructure orientation $\theta$, $\mathbf{c}$ mapping function $\phi_{1}(\boldsymbol{x})$, $\mathbf{d}$ de-homogenized design, e de-homogenized design using

a more uniform spacing as can be seen in Fig. 12e (Groen et al. 2019). It has to be mentioned that different kinds of microstructures (i.e., with non-orthogonal features) can be de-homogenized as well (Geoffroy-Donders 2018; Groen 2019; Kumar and Suresh 2020; Tamijani et al. 2020).

A challenge of the de-homogenization approach is to allow a smooth microstructure orientation throughout the domain. This is especially an issue since the elasticity tensor has a rotational symmetry of $\pi$, i.e., rotating the microstructure $180^{\circ}$ does not affect the properties; however, it complicates the de-homogenization. To circumvent this, Pantz and Trabelsi (2008) proposed a two-field approach to solve for $\phi_{i}(\boldsymbol{x})$. In a different approach, Groen and Sigmund (2018) used an image-based sorting approach, while Allaire et al. (2018) used a discontinuous Galerkin approach to solve for $\phi_{i}(\boldsymbol{x})$. Furthermore, it is known that singularities can occur in the design domain, either caused by the stress field or caused by regularization methods. Hence, the algorithms should be able to handle these singularities. Different approaches to do so are discussed in Pantz and Trabelsi (2010), Geoffroy-Donders (2018), and Stutz et al. (2020). The extension of the approach to 3D requires a solution to the problem that the principal stress directions are not well-ordered in 3D. To circumvent this, GeoffroyDonders et al. (2020) introduced a regularization functional, while Groen et al. (2020) combined a regularization and an image-based sorting approach.

A further challenge is the de-homogenization of rank- $N$ designs optimized for multiple load cases. The non-uniqueness adaptive periodicity approach from (Groen et al. 2019). Reprinted from Groen et al. (2019) with permission from Elsevier

of the optimal microstructures, which allows for an infinite amount of designs, has to be taken into account, since the de-homogenization procedure requires smooth vector fields throughout the design domain. Hence, making sure that the microstructure orientation is continuous throughout $\Omega$, without restricting the performance is a key challenge (Groen 2019).

Above approaches seek a global parameterization of each of the principal stress directions. In a different approach, de-homogenization is cast as finding a quadrilateral (2D) or hexahedral (3D) mesh with each edge being aligned with the optimized stress directions (Wu et al. 2021), borrowing ideas from field-aligned meshing (Jakob et al. 2015; Gao et al. 2017). Instead of decomposing a tensor field into three vector fields, the difference between the orientation of a (locally parameterized) quad/hex element and a stress tensor is measured by comparing all possible perturbations of decomposed directions. The cumulative difference over all elements is minimized by an iterative local updating scheme. This approach is demonstrated in combination with a modified version of the rectangular hole model. As illustrated in Fig. 13a for 2D cases, the unit cell is allowed to rotate $(\theta)$ and elongate independently along each axis $\left(\alpha_{x}\right.$ and $\alpha_{y}$ ). In addition, another design variable (not shown in (a)), similarly used in PAMP (Liu et al. 2008), is introduced to encode whether or not a finite element is filled with lattices. The optimized lattice distribution and the compiled continuous lattice structure are shown in Fig. 13b and c, respectively. The optimized structure possesses spatial

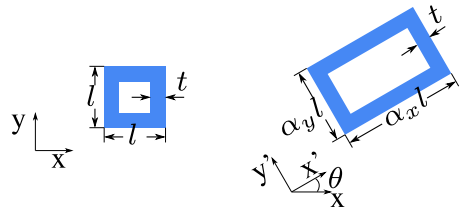

a) Unit cell transformation

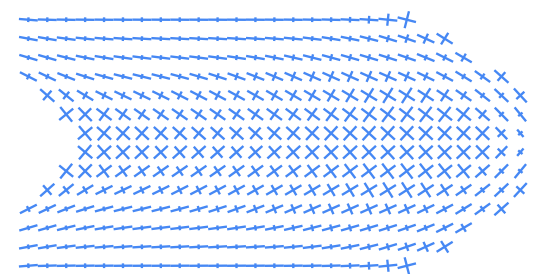

b) Optimized lattice distribution

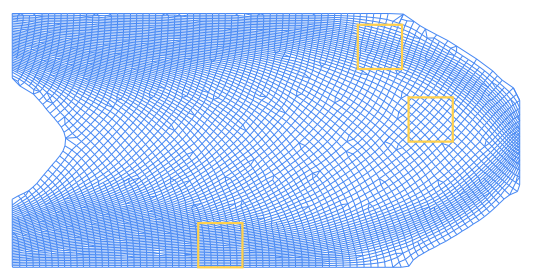

c) Continuous lattice structure
Fig. 13 a The unit cell, a modified version of the rectangular hole model, adapts by rotating and elongation. b The optimized lattice distribution for the Michell cantilever using $15 \%$ solid material. The frames are rotated and elongated according to the optimized fields. c
A continuous lattice structure compiled from $\mathbf{b}$, demonstrating spatial variations in orientation, porosity, and anisotropy (Wu et al. 2021). (C2021 IEEE. Reprinted, with permission, from Wu et al. (2021) 
variations in orientation, porosity, and anisotropy. A 3D example is shown in Fig. 2c.

Yet another mapping strategy is to modify crossing of contour lines of aforementioned $\phi$ fields, connect these by truss or frame elements, and then tune nodal positions and connectivities in a subsequent shape optimization process (Larsen et al. 2018).

\subsection{Restricted unit-cell design}

Predefined cellular structures are beneficial for computational efficiency. However, they may significantly restrict the solution space. To access the full solution space, while avoiding intensive full-scale analysis, an idea is to use a hierarchical formulation. Hierarchical optimization of material and structure dates back to Rodrigues et al. (2002) which was later extended to 3D (Coelho et al. 2008), both using SIMP. Comparable results were reproduced with level sets (Sivapuram et al. 2016). Hierarchical optimization combined with inverse homogenization is also referred to as concurrent (or simultaneous) optimization of structure and material (or microstructure). Like in approaches in other categories, here, a two-scale discretization of the domain is employed. The formulation involves one problem at the global (or macro) scale and many problems at the local (or micro) scale. The global problem determines the macroscopic spatial distribution of homogenized material, and local problems determine microscopic spatial distribution of solid and void phases by optimizing for homogenized properties. The structural equilibrium in the macroscale is in general nonlinear due to the microstructure adaptation (Jog and Haber 1996). A nonlinear resolution framework based on $F E^{2}$ scheme was developed to address this nonlinearity (Xia and Breitkopf 2014). This subsection discusses approaches that prescribe the domain shape of microstructures and/or their orientation.

The microstructural optimization problem (inverse homogenization) should result in single-scale approximations of theoretically optimal rank- $N$ composites. In a recent work, it was found that inverse homogenization at low volume fractions is prone to producing local optima, and a simple mapping approach was suggested to approximate rank-3 laminates (Träff et al. 2019). These mapped microstructures perform relatively close to theoretical energy bounds, and can serve as starting guesses for inverse homogenization problems to achieve performances even closer to the bounds.

In each iteration of a hierarchical solution process, following a solved global problem, the local problems become independent from each other. On the positive side, the independent problems can be solved in parallel by sending sets of local problems to different processors (Coelho et al. 2011). This gains a computational speedup and thus allows solving two-scale problems in 3D. On the other hand, the independent nature of the local problems creates a critical challenge regarding the compatibility of microstructures across the shared boundary. We emphasize that the problem of concern is related to structural properties beyond the disconnected geometry, and thus choose to use compatibility in lieu of connectivity. The compatibility problem arises since disconnections between adjacent microstructures are not captured in the global analysis using homogenized properties (separation of scales). A mechanical indication of compatibility is the discrepancy between the objective (e.g., compliance) evaluated by a full-scale analysis and by an analysis using the homogenized properties (Garner et al. 2019). Some approaches summarized in Section 5.2 for improving compatibility in functionally graded microstructures are also applicable to the hierarchical optimization. For instance, by using extended domains that overlap in local optimizations, the compatibility can be significantly improved, reducing the discrepancy in compliance values between full-scale and homogenization analyses from six orders of magnitude to two (Garner et al. 2019) (see Fig. 14). While this is good progress, a discrepancy of two orders of magnitude is still alarming. To examine the optimality, we can visually compare hierarchically optimized structures with those from full-scale approaches with local volume constraints (Section 3) and de-homogenization approaches (Section 5.3). For a single load, orthogonal microstructures that are individually aligned with principal stress directions are known to be close to optimal. Such orthogonal microstructures are distinct in full-scale approaches with local volume constraints as well as dehomogenization approaches, but are difficult to discover in large regions of hierarchically optimized structures. A reason behind the poor compatibility is that the fixed, axisaligned rectangular domain used in inverse homogenization is incapable of accommodating rotation of these orthogonal microstructures.

Other strategies have been proposed to enhance connectivity. Wang et al. (2017) proposed a shape metamorphosis method based on level-set representations to interpolate a prototype microstructure to generate a family of graded microstructures. The interpolated microstructures are connectable to each other in a natural way since they present similar topological features and material distribution patterns at their edges. Li et al. (2018b) adopted the kinematic constraint approach (Zhou and Li 2008) for level-set-based topology optimization of functionally graded cellular composites hosting auxetic metamaterials. Zhou et al. (2019) proposed a geometric connectivity index upon which constraints were defined and included in the macroscale optimization to improve connectivity. Liu et al. (2020) recently proposed to ensure connectivity between any two types of the microstructures by introducing pre-defined connective 


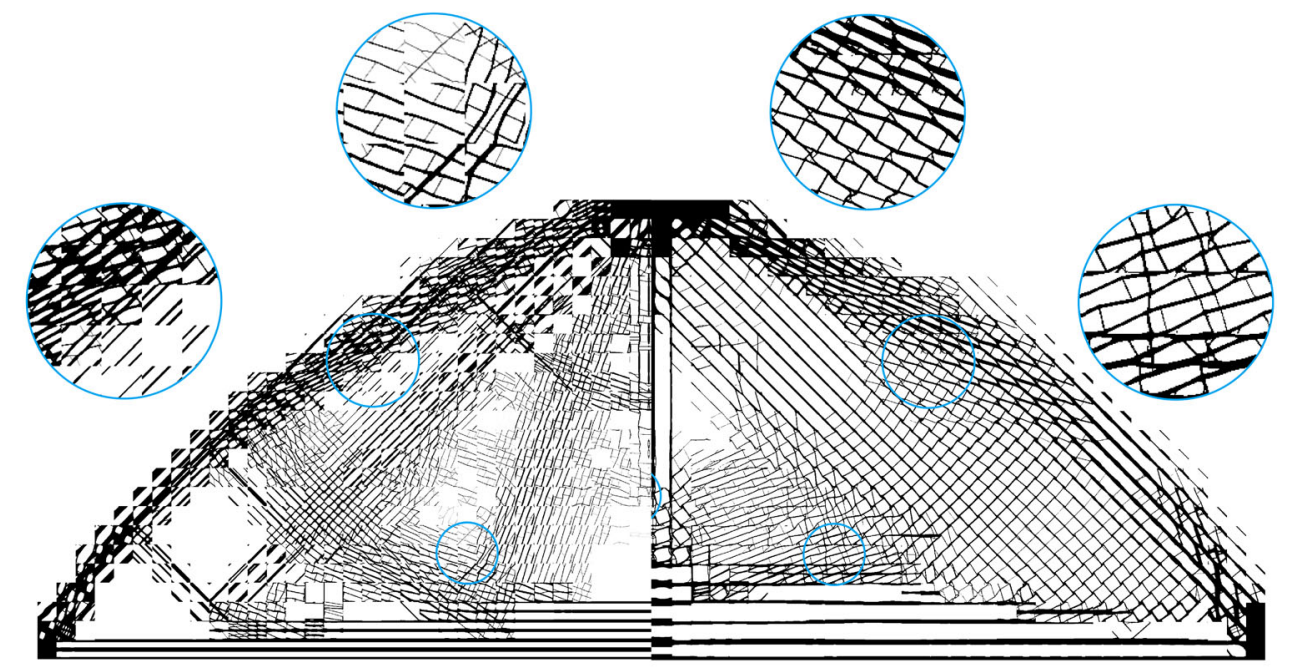

Fig. 14 Illustrative result of hierarchical optimization of material and structure of a simply supported beam, without (left) and with (right) a compound formulation to improve microstructural compatibility (Garner et al. 2019). Half of the optimized structure from each formulation is shown, for compactness. The compliance of the left part by a full-scale analysis and an analysis using homogenized properties is $8.32 \times 10^{13}$ and $6.54 \times 10^{7}$, respectively, while the compliance of the right part is $4.96 \times 10^{9}$ and $8.42 \times 10^{7}$, respectively. Reprinted from Garner et al. (2019) with permission from Elsevier

the context of heat transfer problems. When the location of unique microstructures is not prescribed, it is often cast as a multi-material optimization problem, for which discrete material optimization (Stegmann and Lund 2005) and ordered SIMP interpolation (Zuo and Saitou 2017) are applicable.

As discussed in Section 3, periodic and graded microstructures can also be designed using full-scale approaches. When the structural analysis is performed on the full resolution, a poor connectivity is reflected by a suboptimal objective. Thus, full-scale approaches naturally ensure good connectivity between microstructures or subdomains, at the price of intensive full-scale analyses. Therefore, results from relevant full-scale approaches may serve as a reference for multi-scale approaches of graded microstructures. From the comparison in Fig. 4, it is clear that a logical (but often underreported) consequence of using periodic microstructures with a fixed orientation is a large reduction in stiffness.

\subsection{Unrestricted unit-cell design}

All approaches in the previous section have one thing in common; the shape of the unit-cell domain is rectangular. However, it is very important to acknowledge the effect of the unit-cell domain on the performance. In a large amount of works that consider a single load case, the numerical examples show that the optimized unit cells resemble a rotated version of the microstructure by Bendsøe and Kikuchi (1988) (Fig. 3). However, since the unit-cell domain cannot be rotated, the shape of the microstructures 
is modified to account for a periodic shape, in turn reducing performance. In other words, if the unit-cell design domain was allowed to rotate, a simpler and close to optimal microstructure would have been found.

To investigate the effect of the unit-cell shape and orientation on the performance, Träff et al. (2019) did an extensive comparison of the effect of the starting guess, when inverse homogenization is used to optimize the microstructure performance. They observed that the material design problem is non-unique and highly nonconvex, i.e., different starting guesses result in completely different unit-cell designs and performances. Furthermore, a starting guess of the unit-cell shape and topology based on an optimal rank-3 microstructure resulted in a significantly better performance compared to the use of a rectangular domain. The parallelogram shape of the unit cell, which was optimized as well, allowed for periodicity patterns that cannot be described by a rectangular domain as seen in Fig. 15. Especially for lower volume fractions, Träff et al. (2019) observed that a unit cell with a starting guess and shape based on an optimal rank-3 laminate significantly outperformed (e.g., up to $30 \%$ more efficient) the designs using a traditional rectangular domain. Hence, the choice of the unit-cell domain significantly influences the performance and researchers have to be aware of this when choosing a multi-scale topology optimization algorithm.

Recently, Wang et al. (2019) showed that near-optimal and periodic truss lattice structures could be obtained for multiple load cases by distorting simple Bravais-like lattice structures to a parallelepiped. Besides using a parallelogram in $2 \mathrm{D}$ or a parallelepiped in $3 \mathrm{D}$, one can use many more different types of polygons to solve the homogenization equations (Barbarosie et al. 2017; Podestá et al. 2019). For example, in 2D, a hexagon can be used to describe a periodic isotropic hexagonal microstructure (Sigmund 2000).

From all studies discussed above, we can conclude that performing multi-scale optimization with unit cells
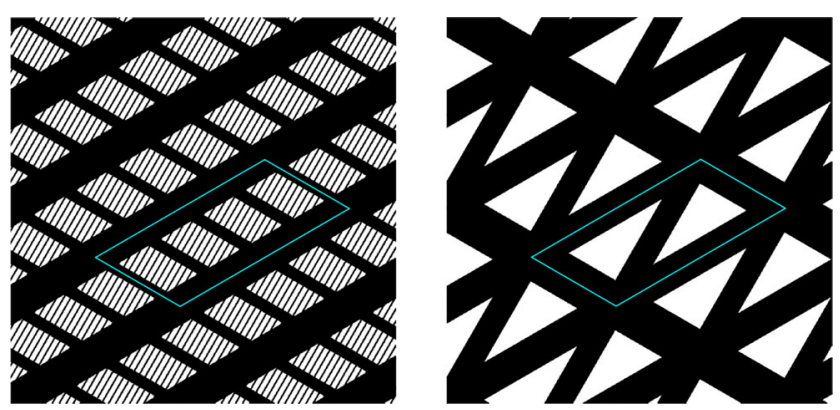

Fig. 15 Left: Rank-3 microstructure with indicated hierarchy. Right: approximated single-scale microstructure using the method by Träff et al. (2019) to indicate different periodicity patterns that cannot be achieved by a rectangular unit cell. Reprinted from Träff et al. (2019) with permission from Springer Nature that are optimized using inverse homogenization on a rectangular domain reduces the optimality. This effect has been acknowledged by Barbarosie and Toader (2014) who combined the optimization of the microstructure topology and shape. This approach can achieve structural designs that are close to optimal; however, an extensive comparison unfortunately has not been performed. To address the large computational cost included in solving the problem, the approach has been parallelized. To the authors' knowledge (and surprise), this is the only work on multi-scale topology optimization that simultaneously addresses the macroscopic design and both unit-cell topology and shape to get as close to the optimal design that can be achieved on a single length scale. Nevertheless, this method does not address the connectivity of the spatially varying unit cells over the design domain. More research has to be done to efficiently blend unit cells of different shapes together without a loss in performance to further advance this method.

\section{Discussion}

\subsection{Motivation}

Two important questions that people working on multi-scale structures should ask themselves are:

- What are the benefits of multi-scale structures?

- What are the expected benefits of multi-scale approaches?

These two questions are related but apparently independent from each other. While multi-scale structures are most often optimized, not surprisingly, by multi-scale approaches, they can also be designed using full-scale approaches with some additional constraints (Section 3). One important motivation of multi-scale topology optimization is to accelerate the computation for optimizing structures at high resolution. Here, it shall be reminded that theoretical stiffnessoptimal structures span multiple scales. A higher resolution discretization enables the appearance of fine geometric details, which may bring the performance of optimized structures closer to theoretical limit. This, however, implies significant computational cost. Thus, multi-scale approaches are introduced for means of acceleration. These include the original hierarchical approach by Rodrigues et al. (2002), de-homogenization approaches (Pantz and Trabelsi 2008; Groen and Sigmund 2018), and works along these directions.

Many multi-scale approaches, while being formulated as an optimization problem (e.g., for maximizing stiffness), restrict the solution space by enforcing one or a few repetitive microstructures that are either predefined or concurrently optimized, with a fixed orientation and/or 
limited density range. This restriction may be beneficial under various considerations, from structural such as buckling strength (Clausen et al. 2016), robustness (Wu et al. 2018), and multi-functionality to operational such as inspection and repair, and from manufacturability over aesthetics to sustainability. In this regard, the microstructures play the role of accounting for these considerations. From a mathematical perspective, it would be interesting to explicitly model these requirements and integrate them in an optimization problem with a high-resolution discretization. This is challenging, since a mathematical model of some of the requirements is not available yet or comes with computational complications. Thus, restricting the solution space by a reduced parameterization can be understood as a strategy to balance the defined objective and various other considerations. A general recommendation is to always motivate use of stiffness suboptimal microstructures. Too many works seem to forget this aspect and show optimized designs that are clearly not optimal with respect to stiffness.

Analogous to technical optimization, nature has been an advocate for multi-scale structures. Hierarchically organized, functionally graded structures can be found in plant and animal bodies such as bamboo and bone (Lakes 1993; Fratzl and Weinkamer 2007). These bio-structures are remarkable from a mechanical perspective while supporting biological functionalities.

\subsection{Evaluation}

Most (if not all) multi-scale approaches make use of homogenization, which assumes separation of scales, i.e., microstructure should be much smaller than the macrostructure. This assumption often becomes invalid when considering the finite resolution of manufacturing processes. A general rule of thumb is that cells should be repeated 5 to 10 times before effective properties can be trusted. This certainly means that commonly seen approaches where one macroscale finite element corresponds to one microstructure cell, where cells may vary between each element, should be used with extreme caution. Interestingly, however, multiscale optimization, where microstructure is appropriately adapted to local stress fields, unimpeded by cell geometry or orientation (c.f., de-homogenization approaches) provides extremely good performances even for quite large periodicities, i.e., with lack of scale separation (see Groen and Sigmund (2018) and Wu et al. (2021)). The reason is that the optimal microstructures ensure purely tensioncompression-dominated deformations at both micro- and macroscale, which homogenization-wise need fewer cell repetitions for accuracy than required for bending or shear dominated deformations. Under all circumstances, it is strongly recommended to verify any assumptions of scale separation with subsequent full-scale analyses. Full-scale analysis of course implies heavy computation. Fortunately, however, recent adoption of advanced linear solvers and parallel computing has partially alleviated this problem (Amir et al. 2014; Aage et al. 2015; Wu et al. 2016a). Validation by full-scale analysis in 2D starts to appear in a handful of recent papers (Groen and Sigmund 2018; Garner et al. 2019; Wu et al. 2021), and should be an integral part of the validation of all multi-scale works.

Optimization in general and multi-scale approaches in particular are about making a delicate balance between conflicting objectives and constraints, and between the quality of results and computational efficiency. In this regard, gains in one aspect are often, understandably, accompanied by losses in other aspects. Both sides are valuable for inspiring future development. These are especially important for researchers who may be less familiar with the topic, e.g., students, application engineers, and colleagues from other disciplines. Therefore, we strongly recommend to include a quantitative comparison (or a discussion if a quantitative analysis is difficult to perform, e.g., regarding aesthetics) when a new approach is introduced. Comparisons can be made on different levels, with non-optimized designs, with optimized mono-scale structures, with designs from alternative multi-scale approaches, etc. In the case of a 2D compliance design subject to a single load case, we recommend to perform a quantitative comparison with what is theoretically possible using rank-2 microstructures. To do this, we provide a Matlab script in Appendix.

\subsection{Extensions, alternative formulations, open questions}

Our review has been focusing on design parameterizations, which are typically demonstrated in compliance minimization under the assumption of small deformations. Accommodating stress constraints, buckling constraints, and geometric and material nonlinearities represents an important and non-trivial next step. Some recent works have started to tackle these challenges.

- Stress constraints. Collet et al. (2018) proposed a formulation for optimizing periodic microstructures with stress constraints, assuming that the classical von Mises stress criterion remains valid at the microscale. Ferrer et al. (2020) proposed a square cell with a superellipsoidal hole, avoiding right angles and thus stress concentration in the classic rectangular hole model. In concurrent optimization of shell and parameterized lattice infill, Yu et al. (2020) proposed using two stress constraints on the macroscale, a von Mises stressbased constraint for the solid shell layer, and a TsaiHill yield criteria-based constraint for the homogenized infill. 
- Buckling, geometric and material nonlinearities, and plasticity. Yuge and Kikuchi (1995) developed elastoplastic finite element analysis based on homogenization to optimize frame structures subjected to plastic deformation. Neves et al. (2002) addressed the problem of determining highly localized buckling modes in perfectly periodic cellular microstructures of infinite extent. Thomsen et al. (2018) proposed a optimization model to predict local and global microstructural buckling, based on which periodic cellular materials are optimized for maximized strength under compressive load. A systematic investigation of the performances of simple and optimized periodic infill structures in terms of finite scale stiffness and buckling was presented by Wang and Sigmund (2020). Strength of common 3D microstructures is treated in Andersen et al. (2021). Furthermore, Bluhm et al. (2020) proposed a framework for benchmarking the ability of periodic microstructures to maintain stiffness under large deformations, accounting in a unified manner both for buckling and softening due to geometric and material nonlinearities.

Going beyond structural mechanics, design of multiscale structures involving other physics and even multiphysics is another important venue to explore. Some of the works along this direction have been mentioned in previous sections when specific multi-scale approaches were introduced. For instance, Das and Sutradhar (2020) presented an extension of the full-scale approach with the local volume constraints (Wu et al. 2018) to optimize heatdissipating structures considering structural and thermal performance. Fluid flow through the pores in lattice structures is another interesting topic, relevant for design of actuators (Andreasen and Sigmund 2011) and biomedical implants (Challis et al. 2012). Furthermore, as discussed in Section 2.3, inverse homogenization has been used to design metamaterials with extreme or counter-intuitive physical properties such as a negative Poisson's ratio and negative thermal expansion (Sigmund and Torquato 1996). Designing multi-scale structures composed of such metamaterials is fascinating. It can potentially open innovative application areas in shape-morphing products, soft robots, and 4D printing (i.e., with an extra dimension of transformation over time).

Optimized structures from multi-scale approaches typically have two scales. It is worth mentioning that bucklingenhanced microstructures themselves exhibit two (or more) hierarchical scales (Thomsen et al. 2018). Integrating them in macroscale structural optimization would effectively lead to three-scale structures. The microstructures in two-scale approaches are normally defined in a uniform discretization of the macro domain, and thus have the same spatial extent.
Wu et al. (2016b) proposed a full-scale approach to design octree-tree like structures, where the cells exhibit spatially varying sizes. This allows to achieve a large range of variations in porosity and pore sizes. The discrete problem of hierarchical subdivision was later reformulated by continuous variables to facilitate gradient-based optimization $(\mathrm{Wu}$ 2018).

Microstructures are typically defined and analyzed based on a Cartesian grid discretization with a fixed orientation. Approximating free-form macroscale shape by square or cubic microstructures leads to staircasing on the boundaries. This artifact may be less of a concern when the macroscale domain is orders of magnitude larger than the microstructure size. However, with the limited resolution of manufacturing processes, the effects of this artifact may be non-negligible, both visually and mechanically. Conforming quad/hex meshing can be an alternative for constructing a boundary-aligned grid (Wu et al. 2021). However, mapping square or cubic microstructures into irregular grids may introduce error in mechanical properties. Note that homogenization assumes a repeatable domain, and thus direct homogenization over irregular cells seems not possible.

As stated in the Introduction section, much of the recent interest in designing multi-scale structures is triggered by contemporary advances in additive manufacturing (AM). While being able to produce highly complex shapes and even structural features spanning seven orders of magnitude (Zheng et al. 2016), existing AM processes are not free from manufacturability and post-processing requirements. Structural design thus needs to consider, e.g., a minimum feature size, self-supportingness (i.e., free of critical overhang), accessibility for removing unsolidified powder or resin, and auxiliary support structure (Liu et al. 2018a). In the context of multi-scale design, these requirements (or some of them) can be satisfied by carefully selecting unit cells, e.g., beam-like lattice cells (see prints in Fig. 2) or self-supporting rhombic cells (Wu et al. 2016b). Stiffness optimal rank- $N$ laminates and closed-walled cells with stiffness close to theoretical bounds (Sigmund et al. 2016) may, depending on process, be less favorable than open-walled cells regarding the removal of unsolidified powder or resin. Along with developments of advanced manufacturing technologies, design for manufacturability will continue to be an important topic in (multi-scale) topology optimization.

The design of multi-scale structures is a topic of interest in multiple other disciplines apart from applied mathematics, computational mechanics, and mechanical engineering. We envisage that cross-pollination with material science (e.g., multi-scale materials modelling (van der Giessen et al. 2020)) as well as geometry modelling and processing (e.g., texture synthesis (Dumas et al. 2015)) will further advance 
optimization and inverse design of multi-scale structures. The cross-pollination is actually already benefiting the field. For instance, the conformal-like mapping and field-aligned meshing have been used for de-homogenization.

\section{Conclusion}

We have reviewed the development of multi-scale topology optimization, from its inception to the current state-of-theart. Existing approaches are classified into full-scale and multi-scale approaches, according to whether or not the separation of length scales is assumed in the modelling. Full-scale approaches control structural details by imposing, e.g., pattern repetition and local volume constraints, while performing structural analysis on the fine scale, which is computation-intensive. Multi-scale approaches reduce computation by using analytical or numerical homogenization, under the assumption of separation of length scales, which may give rise to compatibility issues. These approaches are categorized in this review based on the parameterization of the micro- and macroscales.

The development in this field is exciting. Innovative approaches and applications continue to appear. To construct an objective understanding of multi-scale structures and multi-scale optimization approaches, we make the following recommendations:

- Since the assumption of separation of length scales may not be respected in the optimized multi-scale structures, structural performance evaluated by homogenization may not faithfully represent reality. It is thus strongly recommended to compare with full-scale analysis when homogenization is used in the optimization approach.

- Many parameterizations have been introduced for designing multi-scale structures while reducing the gap between full-scale and homogenization-based analyses. These parameterizations may (severely) limit the achievable structural objective. It is thus also recommended to compare with standard mono-scale approaches under a comparable computation time, when investigating homogenization-based methods.

- Multi-scale structures hold the promise of achieving superior performance while being intrinsically lightweight, robust, and multi-functional. The true benefits of novel multi-scale structures need to be validated, numerically, and/or experimentally.

\section{Appendix : MATLAB code topRank2}

Together with this review, we present a MATLAB code for the topology optimization of $2 \mathrm{D}$ structures subject to a single load case using optimal rank-2 microstructures consisting of solid material (with stiffness $E^{+}$) and void. The code is based on the MATLAB code by Andreassen et al. (2011), and we will therefore only discuss the differences. The most obvious difference is the use of a rank-2 material model (see Fig. 5 for the visualization of a unit cell). The effective material property can be analytically derived using the homogenization equation (see, e.g., Allaire (2002) and Bendsøe and Sigmund (2004)), and can be summarized in Voigt notation as,

$$
\begin{aligned}
E^{H}\left(\mu_{1}, \mu_{2}, \theta\right)= & \frac{E^{-}}{1-v^{2}}\left[\begin{array}{ccc}
1 & v & 0 \\
v & 1 & 0 \\
0 & 0 & \frac{1-v}{2}
\end{array}\right]+\frac{E^{+}}{1-\mu_{2}+\mu_{1} \mu_{2}(1-v)} \\
& \mathbf{R}^{T}(\theta)\left[\begin{array}{ccc}
\mu_{1} & \mu_{1} \mu_{2} v & 0 \\
\mu_{1} \mu_{2} v & \mu_{2}\left(1-\mu_{2}+\mu_{1} \mu_{2}\right) & 0 \\
0 & 0 & 0
\end{array}\right] \mathbf{R}(\theta)
\end{aligned}
$$

Since a rank-2 microstructure consisting of solid material and void contains no stiffness against shearing, a small isotropic background stiffness $E^{-}$is added to make this material model work stable on a finite mesh. To minimize the effect of this background stiffness and avoid getting stuck in local minima, we start with $E^{-}=0.1 E^{+}$and gradually reduce every 50 iterations until $E^{-}=10^{-6} E^{+}$. For efficient assembly of the stiffness matrix, we preintegrated 6 element matrices, i.e., one for each unique index of the rotated elasticity tensor. As is discussed by Pedersen (1989), a microstructure is optimally aligned with the principal stresses/strains. Therefore, we update the microstructure orientation $(\theta)$ during each design iteration based on the principal stress directions. Subsequently, we update the relative layer widths $\left(\mu_{i}\right)$ based on the gradients using the optimality criterion approach. Finally, it should be noted that we use the density filter to avoid the formation of checkerboard-like patterns that are artificially stiff. Similar to the 88-line MATLAB code, the default design problem is the half MBB-beam, where the compliance is minimized subject to an upper bound on the volume fraction of the stiff material. The code can be called as follows:

$$
\text { toprank2(nelX,nelY,rMin,volFrac) }
$$

where nelX and nelY are the number of bi-linear elements in $\mathrm{x}$ - and $\mathrm{y}$-direction, respectively, rMin is the filter radius in element length $h$ used for the density filter, and vol Frac is the volume fraction that the stiff material is allowed to use.

Supplementary Information The online version contains supplementary material available at https://doi.org/10.1007/s00158-021-02881-8.

Funding O. Sigmund and J. Groen were supported by the Villum Fonden through the Villum Investigator Project "InnoTop." 


\section{Declarations}

Conflict of interest The authors declare no competing interests.

Replication of results Results presented in this review are based on published methods in the literature. We refer the reader to the original work for more details of each method. Important details for replicating the results have been described in the manuscript. The code for examples shown in Figs. 4 and 8 is available on request from the corresponding author.

Open Access This article is licensed under a Creative Commons Attribution 4.0 International License, which permits use, sharing, adaptation, distribution and reproduction in any medium or format, as long as you give appropriate credit to the original author(s) and the source, provide a link to the Creative Commons licence, and indicate if changes were made. The images or other third party material in this article are included in the article's Creative Commons licence, unless indicated otherwise in a credit line to the material. If material is not included in the article's Creative Commons licence and your intended use is not permitted by statutory regulation or exceeds the permitted use, you will need to obtain permission directly from the copyright holder. To view a copy of this licence, visit http://creativecommons. org/licenses/by/4.0/

\section{References}

Aage N, Andreassen E, Lazarov BS (2015) Topology optimization using PETSc: an easy-to-use, fully parallel, open source topology optimization framework. Struct Multidiscip Optim 51(3):565572. https://doi.org/10.1007/s00158-014-1157-0

Alexandersen J, Lazarov BS (2015) Topology optimisation of manufacturable microstructural details without length scale separation using a spectral coarse basis preconditioner. Comput Methods Appl Mech Eng 290:156-182. https://doi.org/10.1016/j.cma.2015.02.028

Allaire G (2002) Shape optimization by the homogenization method, vol 146. Springer Science \& Business Media

Allaire G, Aubry S (1999) On optimal microstructures for a plane shape optimization problem. Struct Optim 17(2):86-94. https://doi.org/10.1007/BF01195933

Allaire G, Kohn R (1993) Optimal-design for minimum weight and compliance in plane-stress using extremal microstructures. Eur J Mech A-Solid 12(6):839-878

Allaire G, Belhachmi Z, Jouve F (1996) The homogenization method for topology and shape optimization. single and multiple loads case. Revue Européenne des Éléments Finis 5(5-6):649-672. https://doi.org/10.1080/12506559.1996.10511241

Allaire G, Bonnetier E, Francfort G, Jouve F (1997) Shape optimization by the homogenization method. Numer Math 76(1):27-68. https://doi.org/10.1007/s002110050253

Allaire G, Jouve F, Toader AM (2004) Structural optimization using sensitivity analysis and a level-set method. J Comput Phys 194(1):363-393. https://doi.org/10.1016/j.jcp.2003.09.032

Allaire G, Geoffroy-Donders P, Pantz O (2018) Topology optimization of modulated and oriented periodic microstructures by the homogenization method. Computers \& Mathematics with Applications. https://doi.org/10.1016/j.camwa.2018.08.007

Almeida SR, Paulino GH, Silva EC (2010) Layout and material gradation in topology optimization of functionally graded structures: a global-local approach. Struct Multidiscip Optim 42(6):855-868. https://doi.org/10.1007/s00158-010-0514-x
Amir O, Aage N, Lazarov BS (2014) On multigrid-cg for efficient topology optimization. Struct Multidiscip Optim 49(5):815-829. https://doi.org/10.1007/s00158-013-1015-5

Andersen MN, Wang F, Sigmund O (2021) On the competition for ultimately stiff and strong architected materials. Mater Des 198:109356. https://doi.org/10.1016/j.matdes.2020.109356

Andreasen CS, Sigmund O (2011) Saturated poroelastic actuators generated by topology optimization. Struct Multidiscip Optim 43(5):693-706. https://doi.org/10.1007/s00158-010-0597-4

Andreassen E (2015) Optimal design of porous materials. PhD thesis

Andreassen E, Andreasen CS (2014) How to determine composite material properties using numerical homogenization. Comput Mater Sci 83:488-495. https://doi.org/10.1016/j.commatsci.2013.09.006

Andreassen E, Clausen A, Schevenels M, Lazarov BS, Sigmund O (2011) Efficient topology optimization in MATLAB using 88 lines of code. Struct Multidiscip Optim 43(1):1-16. https://doi.org/10.1007/s00158-010-0594-7

Andreassen E, Lazarov BS, Sigmund O (2014) Design of manufacturable 3d extremal elastic microstructure. Mech Mater 69(1):110. https://doi.org/10.1016/j.mechmat.2013.09.018

Avellaneda M (1987) Optimal bounds and microgeometries for elastic two-phase composites. SIAM J Appl Math 47(6):1216-1228. https://doi.org/10.1137/0147082

Barbarosie C, Toader AM (2014) Optimization of bodies with locally periodic microstructure by varying the periodicity pattern. Netw Heterog Media 9(3):433-451. https://doi.org/10.3934/nhm.2014.9.433

Barbarosie C, Tortorelli DA, Watts S (2017) On domain symmetry and its use in homogenization. Comput Methods Appl Mech Eng 320:1-45. https://doi.org/10.1016/j.cma.2017.01.009

Beghini LL, Beghini A, Katz N, Baker WF, Paulino GH (2014) Connecting architecture and engineering through structural topology optimization. Eng Struct 59:716-726. https://doi.org/10.1016/j.engstruct.2013.10.032

Bendsøe M, Sigmund O (2004) Topology optimization: theory, methods, and applications. Springer, Berlin

Bendsøe MP (1989) Optimal shape design as a material distribution problem. Struct Optim 1(4):193-202. https://doi.org/10.1007/BF01650949

Bendsøe MP, Kikuchi N (1988) Generating optimal topologies in structural design using a homogenization method. Comput Methods Appl Mech Eng 71(2):197-224. https://doi.org/10.1016/0045-7825(88)90086-2

Bendsøe MP, Sigmund O (1999) Material interpolation schemes in topology optimization. Arch Appl Mech 69(9-10):635-654. https://doi.org/10.1007/s004190050248

Bendsøe MP, Guedes JM, Haber RB, Pedersen P, Taylor JE (1994) An analytical model to predict optimal material properties in the context of optimal structural design. J Appl Mech 61(4):930. https://doi.org/10.1115/1.2901581

Bensoussan A, Lions JL, Papanicolaou G (1978) Asymptotic analysis for periodic structures (studies in mathematics and its applications). Elsevier Science Ltd

Bluhm GL, Sigmund O, Wang F, Poulios K (2020) Nonlinear compressive stability of hyperelastic $2 \mathrm{~d}$ lattices at finite volume fractions. J Mech Phys Solids 137:103851. https://doi.org/10.1016/j.jmps.2019.103851

Brackett D, Ashcroft I, Hague R (2011) Topology optimization for additive manufacturing. In: Proceedings of the solid freeform fabrication symposium, Austin, TX, vol 1, pp 348-362

Cadman JE, Zhou S, Chen Y, Li Q (2013) On design of multifunctional microstructural materials. J Mater Sci 48(1):51-66. https://doi.org/10.1007/s10853-012-6643-4 
Cang R, Yao H, Ren Y (2019) One-shot generation of near-optimal topology through theory-driven machine learning. Comput Aided Des 109:12-21. https://doi.org/10.1016/j.cad.2018.12.008

Carstensen JV, Guest JK (2018) Projection-based two-phase minimum and maximum length scale control in topology optimization. Struct Multidiscip Optim 58(5):1845-1860. https://doi.org/10.1007/s00158-018-2066-4

Challis VJ, Guest JK, Grotowski JF, Roberts AP (2012) Computationally generated cross-property bounds for stiffness and fluid permeability using topology optimization. Int J Solids Struct 49(23):3397-3408. https://doi.org/10.1016/j.ijsolstr.2012.07.019

Cheng KT (1981) On non-smoothness in optimal design of solid, elastic plates. Int J Solids Struct 17(8):795-810. https://doi.org/10.1016/0020-7683(81)90089-5

Cheng KT, Olhoff N (1981) An investigation concerning optimal design of solid elastic plates. Int J Solids Struct 17(3):305-323. https://doi.org/10.1016/0020-7683(81)90065-2

Cherkaev A, Palais R (1996) Optimal design of three-dimensional axisymmetric elastic structures. Struct Optim 12(1):35-45. https://doi.org/10.1007/bf01270442

Cherkaev A, Krog L, Kucuk I (1998) Stable optimal design of twodimensional elastic structures. Control Cybern 27(2):265-282

Christiansen RE, Wang F, Sigmund O, Stobbe S (2019) Designing photonic topological insulators with quantum-spin-hall edge states using topology optimization. Nanophotonics 8(8):1363-1369. https://doi.org/10.1515/nanoph-2019-0057

Clausen A, Aage N, Sigmund O (2015a) Topology optimization of coated structures and material interface problems. Comput Methods Appl Mech Eng 290:524-541. https://doi.org/10.1016/j.cma.2015.02.011

Clausen A, Wang F, Jensen JS, Sigmund O, Lewis JA (2015b) Topology optimized architectures with programmable poisson's ratio over large deformations. Adv Mater 27(37):5523-5527. https://doi.org/10.1002/adma.201502485

Clausen A, Aage N, Sigmund O (2016) Exploiting additive manufacturing infill in topology optimization for improved buckling load. Engineering 2(2):250-257. https://doi.org/10.1016/J.ENG.2016.02.006

Coelho P, Cardoso J, Fernandes P, Rodrigues H (2011) Parallel computing techniques applied to the simultaneous design of structure and material. Adv Eng Softw 42(5):219-227. https://doi.org/10.1016/j.advengsoft.2010.10.003

Coelho PG, Fernandes PR, Guedes JM, Rodrigues HC (2008) A hierarchical model for concurrent material and topology optimisation of three-dimensional structures. Struct Multidiscip Optim 35(2):107-115. https://doi.org/10.1007/s00158-007-0141-3

Collet M, Noël L, Bruggi M, Duysinx P (2018) Topology optimization for microstructural design under stress constraints. Struct Multidiscip Optim 58(6):2677-2695. https://doi.org/10.1007/s00158-018-2045-9

Cramer AD, Challis VJ, Roberts AP (2016) Microstructure interpolation for macroscopic design. Struct Multidiscip Optim 53(3):489500. https://doi.org/10.1007/s00158-015-1344-7

Czarnecki S, Lewiński T (2006) Shaping the stiffest three-dimensional structures from two given isotropic materials. Comput Assist Mech Eng Sci 13(1):53-83

Das S, Sutradhar A (2020) Multi-physics topology optimization of functionally graded controllable porous structures: application to heat dissipating problems. Mater Des 193:108775. https://doi.org/10.1016/j.matdes.2020.108775

Deaton JD, Grandhi RV (2014) A survey of structural and multidisciplinary continuum topology optimization: post 2000. Struct Multidiscip Optim 49(1):1-38. https://doi.org/10.1007/s00158-013-0956-z
Deng J, Chen W (2017) Concurrent topology optimization of multiscale structures with multiple porous materials under random field loading uncertainty. Struct Multidiscip Optim 56(1):1-19. https://doi.org/10.1007/s00158-017-1689-1

Díaz A, Lipton R (1997) Optimal material layout for 3d elastic structures. Struct Optim 13(1):60-64. https://doi.org/10.1007/bf 01198376

Díaz AR, Lipton R (2000) Optimal material layout in threedimensional elastic structures subjected to multiple loads. Mech Struct Mach 28(2-3):219-236. https://doi.org/10.1081/sme-10010 0619

Díaz AR, Lipton R, Soto CA (1995) A new formulation of the problem of optimum reinforcement of reissner-mindlin plates. Comput Methods Appl Mech Eng 123(1):121-139. https://doi.org/10.1016/0045-7825(94)00777-K

Dong G, Tang Y, Zhao YF (2019) A 149 line homogenization code for three-dimensional cellular materials written in MATLAB. J Eng Mater Technol 141(1):011005. https://doi.org/10.1115/1.4040555

Dou S (2020) A projection approach for topology optimization of porous structures through implicit local volume control. Struct Multidiscip Optim 1-16 https://doi.org/10.1007/s00158-020-025 $39-x$

Du Z, Zhou XY, Picelli R, Kim HA (2018) Connecting microstructures for multiscale topology optimization with connectivity index constraints. J Mech Des 140(11):12. https://doi.org/10.1115/1. 4041176

Dumas J, Lu A, Lefebvre S, Wu J, Dick C (2015) By-Example Synthesis of structurally sound patterns. ACM Trans Graph 34(4):12. https://doi.org/10.1145/2766984

Ferrari F, Sigmund O (2020) A new generation 99 line Matlab code for compliance topology optimization and its extension to 3D. arXiv:200505436

Ferrer A, Georoy-Donders P, Allaire G (2020) Stress minimization for lattice structures. Part I: micro-structure design, https://hal. archives-ouvertes.fr/hal-02889138, working paper or preprint

Fleury C (1993) Mathematical programming methods for constrained optimization: dual methods. In: Kamat P (ed) Structural optimization: status and promise, progress in astronautics and aeronautics, vol 150, AIAA, chap, 7, pp 123-150. https://doi.org/10.2514/5.9781600866234.0123.0150

Francfort GA, Murat F (1986) Homogenization and optimal bounds in linear elasticity. Arch Ration Mech Anal 94(4):307-334. https://doi.org/10.1007/BF00280908

Fratzl P, Weinkamer R (2007) Nature's hierarchical materials. Prog Mater Sci 52(8):1263-1334. https://doi.org/10.1016/j.pmatsci.2007.06. 001

Fujii D, Chen BC, Kikuchi N (2001) Composite material design of two-dimensional structures using the homogenization design method. Int J Numer Methods Eng 50(9):2031-2051. https://doi.org/10.1002/nme.105

Gao X, Jakob W, Tarini M, Panozzo D (2017) Robust hexdominant mesh generation using field-guided polyhedral agglomeration. ACM Trans Graph 36(4):114:1-114:13. https://doi.org/10.1145/3072959.3073676

Garner E, Kolken HM, Wang CC, Zadpoor AA, Wu J (2019) Compatibility in microstructural optimization for additive manufacturing. Addit Manuf 26:65-75. https://doi.org/10.1016/j.addma.2018.12.007

Geoffroy-Donders P (2018) Homogenization method for topology optimization of structures built with lattice materials. Theses, Ecole Polytechnique

Geoffroy-Donders P, Allaire G, Pantz O (2020) 3-d topology optimization of modulated and oriented periodic microstructures by the homogenization method. J Computat Phys 401:108994. https://doi.org/10.1016/j.jcp.2019.108994 
van der Giessen E, Schultz PA, Bertin N, Bulatov VV, Cai W, Csányi G, Foiles SM, Geers MG, González C, Hütter $M$ et al (2020) Roadmap on multiscale materials modeling. Modell Simul Mater Sci Eng 28(4):043001. https://doi.org/10.1088/1361-651X/ab7150

Groen JP (2019) Multi-scale design methods for topology optimization. $\mathrm{PhD}$ thesis

Groen JP, Sigmund O (2018) Homogenization-based topology optimization for high-resolution manufacturable microstructures. Int $\mathbf{J}$ Numer Methods Eng 113(8):1148-1163. https://doi.org/10.1002/ nme. 5575

Groen JP, Wu J, Sigmund O (2019) Homogenization-based stiffness optimization and projection of $2 \mathrm{~d}$ coated structures with orthotropic infill. Comput Methods Appl Mech Eng 349:722742

Groen JP, Stutz FC, Aage N, Bærentzen JA, Sigmund O (2020) Dehomogenization of optimal multi-scale $3 \mathrm{~d}$ topologies. Comput Methods Appl Mech Eng 364:112979. https://doi.org/10.1016/j. cma.2020.112979

Guedes JM, Kikuchi N (1990) Preprocessing and postprocessing for materials based on the homogenization method with adaptive finite element methods. Comput Methods Appl Mech Eng 83(2):143-198. https://doi.org/10.1016/0045-7825(90)90148-F

Guedes JM, Rodrigues HC, Bendsøe MP (2003) A material optimization model to approximate energy bounds for cellular materials under multiload conditions. Struct Multidiscip Optim 25(5-6):446-452. https://doi.org/10.1007/s00158-003-0305-8

Guest JK (2009) Imposing maximum length scale in topology optimization. Struct Multidiscip Optim 37(5):463-473. https://doi.org/10.1007/s00158-008-0250-7

Guest JK, Prévost JH, Belytschko T (2004) Achieving minimum length scale in topology optimization using nodal design variables and projection functions. Int J Numer Methods Eng 61(2):238254. https://doi.org/10.1002/nme.1064

Guo X, Zhang W, Zhong W (2014) Doing topology optimization explicitly and geometrically-a new moving morphable components based framework. J Appl Mech 81(8):12. https://doi.org/10.1115/ 1.4027609

Guo X, Zhao X, Zhang W, Yan J, Sun G (2015) Multiscale robust design and optimization considering load uncertainties. Comput Methods Appl Mech Eng 283:994-1009. https://doi.org/10.1016/j.cma.2014.10.014

Hammer V, Bendsøe M, Lipton R, Pedersen P (1997) Parametrization in laminate design for optimal compliance. Int J Solids Struct 34(4):415-434. https://doi.org/10.1016/s0020-7683(96)00023-6

Hashin Z, Shtrikman S (1963) A variational approach to the theory of the elastic behaviour of multiphase materials. J Mech Phys Solids 11(2):127-140. https://doi.org/10.1016/0022-5096(63)90060-7

$\mathrm{Hu}$ J, Li M, Yang X, Gao S (2020) Cellular structure design based on free material optimization under connectivity control. Comput Aided Des 127:102854. https://doi.org/10.1016/j.cad.2020.102854

Huang X, Xie Y (2008) Optimal design of periodic structures using evolutionary topology optimization. Struct Multidiscip Optim 36(6):597-606. https://doi.org/10.1007/s00158-007-0196-1

Huang X, Zhou S, Xie Y, Li Q (2013) Topology optimization of microstructures of cellular materials and composites for macrostructures. Comput Mater Sci 67:397-407. https://doi.org/10.1016/j.commatsci.2012.09.018

Imediegwu C, Murphy R, Hewson R, Santer M (2019) Multiscale structural optimization towards three-dimensional printable structures. Struct Multidiscip Optim 60(2):513-525. https://doi.org/10.1007/s00158-019-02220-y

Jakob W, Tarini M, Panozzo D, Sorkine-Hornung O (2015) Instant field-aligned meshes. ACM Trans Graph 34(6):15. https://doi.org/ https://doi.org/10.1145/2816795.2818078
Jansen M, Lombaert G, Schevenels M, Sigmund O (2014) Topology optimization of fail-safe structures using a simplified local damage model. Struct Multidiscip Optim 49(4):657-666. https://doi.org/10.1007/s00158-013-1001-y

Jensen J, Sigmund O (2011) Topology optimization for nano-photonics. Laser Photonics Rev 5(2):308-321. https://doi.org/10.1002/lpor.201000014

Jog CS, Haber RB (1996) Stability of finite element models for distributed-parameter optimization and topology design. Comput Methods Appl Mech Eng 130(3-4):203-226. https://doi.org/10.1016/0045-7825(95)00928-0

Kohn RV, Strang G (1986) Optimal design and relaxation of variational problems, i. Commun Pur Appl Math 39(1):113-137. https://doi.org/10.1002/cpa.3160390107

Krog LA, Olhoff N (1999) Optimum topology and reinforcement design of disk and plate structures with multiple stiffness and eigenfrequency objectives. Comput Struct 72(4-5):535-563. https://doi.org/10.1016/s0045-7949(98)00326-5

Kumar T, Suresh K (2020) A density-and-strain-based k-clustering approach to microstructural topology optimization. Struct Multidiscip Optim 61(4):1399-1415. https://doi.org/10.1007/s00158-019-02422-4

Lakes R (1993) Materials with structural hierarchy. Nature 361(6412):511-515. https://doi.org/10.1038/361511a0

Larsen SD, Sigmund O, Groen JP (2018) Optimal truss and frame design from projected homogenization-based topology optimization. Struct Multidiscip Optim 57(4):1461-1474. https://doi.org/10.1007/s00158-018-1948-9

Lazarov BS, Wang F (2017) Maximum length scale in density based topology optimization. Comput Methods Appl Mech Eng 318:826-844. https://doi.org/10.1016/j.cma.2017.02.018

Lévy B, Petitjean S, Ray N, Maillot J (2002) Least squares conformal maps for automatic texture atlas generation. ACM Trans Graph 21(3):10. https://doi.org/10.1145/566654.566590

Li D, Liao W, Dai N, Dong G, Tang Y, Xie YM (2018a) Optimal design and modeling of gyroid-based functionally graded cellular structures for additive manufacturing. Comput Aided Des 104:8799. https://doi.org/10.1016/j.cad.2018.06.003

Li H, Luo Z, Gao L, Walker P (2018b) Topology optimization for functionally graded cellular composites with metamaterials by level sets. Comput Methods Appl Mech Eng 328:340-364. https://doi.org/10.1016/j.cma.2017.09.008

Li H, Gao L, Li H, Tong H (2020) Spatial-varying multi-phase infill design using density-based topology optimization. Comput Methods Appl Mech Eng 372:113354. https://doi.org/10.1016/j.cma.2020.113354

Lipton R (1994a) On optimal reinforcement of plates and choice of design parameters. Control Cybern 23(3):481-493

Lipton R (1994b) A saddle-point theorem with application to structural optimization. J Optim Theory Appl 81(3):549-568. https://doi.org/10.1007/bf02193100

Liu J, Gaynor AT, Chen S, Kang Z, Suresh K, Takezawa A, Li L, Kato J, Tang J, Wang CCL, Cheng L, Liang X, To AC (2018a) Current and future trends in topology optimization for additive manufacturing. Struct Multidiscip Optim 57(6):24572483. https://doi.org/10.1007/s00158-018-1994-3

Liu L, Yan J, Cheng G (2008) Optimum structure with homogeneous optimum truss-like material. Comput Struct 86(13):1417-1425. https://doi.org/10.1016/j.compstruc.2007.04.030

Liu P, Kang Z, Luo Y (2020) Two-scale concurrent topology optimization of lattice structures with connectable microstructures. Addit Manuf 36:101427. https://doi.org/10.1016/j.addma.2020.101427

Liu Y, Li Z, Wei P, Wang W (2018b) Parameterized level-set based topology optimization method considering symmetry and pattern repetition constraints. Comput Methods Appl Mech Eng 340:1079-1101. https://doi.org/10.1016/j.cma.2018.04.034 
Luo Y, Li Q, Liu S (2019) A projection-based method for topology optimization of structures with graded surfaces. Int $\mathrm{J}$ Numer Methods Eng 118(11):654-677. https://doi.org/10.1002/nme.6031

Lurie KA, Cherkaev AV (1984) G-closure of a set of anisotropically conducting media in the two-dimensional case. J Optim Theory Appl 42(2):283-304. https://doi.org/10.1007/BF00934300

Michell A (1904) The limits of economy of material in frame-structures. The London Edinburgh, and Dublin Philosophical Magazine and Journal of Science 8(47):589-597. https://doi.org/10.1080/14786440409463229

Milton G, Harutyunyan D, Briane M (2017) Towards a complete characterization of the effective elasticity tensors of mixtures of an elastic phase and an almost rigid phase. Math Mech Complex Syst 5(1):95-113. https://doi.org/10.2140/memocs.2017.5.95

Milton GW (1986) Modelling the properties of composites by laminates. In: Ericksen JL, Kinderlehrer D, Kohn R, Lions JL (eds) Homogenization and effective moduli of materials and media. Springer, New York, pp 150-174. https://doi.org/10.1007/978-1-4613-8646-9_

Milton GW (2002) The Theory of Composites. Cambridge University Press, Cambridge. https://doi.org/10.1017/cbo9780511613357

Milton GW (2016) Extending the theory of composites to other areas of science. Milton-Patton Publishers

Mlejnek H (1992) Some aspects of the genesis of structures. Struct Optim 5(1-2):64-69. https://doi.org/10.1007/BF01744697

Neves MM, Sigmund O, Bendsøe MP (2002) Topology optimization of periodic microstructures with a penalization of highly localized buckling modes. Int J Numer Methods Eng 54(6):809-834. https://doi.org/10.1002/nme.449

Niordson F (1983) Optimal design of elastic plates with a constraint on the slope of the thickness function. Int J Solids Struct 19(2):141151. https://doi.org/10.1016/0020-7683(83)90005-7

Niu B, Yan J, Cheng G (2009) Optimum structure with homogeneous optimum cellular material for maximum fundamental frequency. Struct Multidiscip Optim 39(2):115. https://doi.org/10.1007/s00158-008-0334-4

Norato J, Haber R, Tortorelli D, Bendsøe MP (2004) A geometry projection method for shape optimization. Int J Numer Methods Eng 60(14):2289-2312. https://doi.org/10.1002/nme.1044

Norris A (1985) A differential scheme for the effective moduli of composites. Mech Mater 4(1):1-16. https://doi.org/10.1016/0167-6636(85)90002-x

Olhoff N, Rønholt E, Scheel J (1998) Topology optimization of threedimensional structures using optimum microstructures. Struct Optim 16(1):1-18. https://doi.org/10.1007/bf01213995

Osanov M, Guest JK (2016) Topology optimization for architected materials design. Annu Rev Mater Res 46(1):211-233. https://doi.org/10.1146/annurev-matsci-070115-031826

Panesar A, Abdi M, Hickman D, Ashcroft I (2018) Strategies for functionally graded lattice structures derived using topology optimisation for additive manufacturing. Addit Manuf 19:81-94. https://doi.org/10.1016/j.addma.2017.11.008

Pantz O, Trabelsi K (2008) A post-treatment of the homogenization method for shape optimization. SIAM J Control Optim 47(3):1380-1398. https://doi.org/10.1137/070688900

Pantz O, Trabelsi K (2010) Construction of minimization sequences for shape optimization. In: 15th International conference on methods and models in automation and robotics (MMAR), pp 278-283. https://doi.org/10.1109/MMAR.2010.5587222

Pedersen P (1989) On optimal orientation of orthotropic materials. Struct Optim 1(2):101-106. https://doi.org/10.1007/BF01637666

Pizzolato A, Sharma A, Maute K, Sciacovelli A, Verda V (2019) Multi-scale topology optimization of multi-material structures with controllable geometric complexity — applications to heat transfer problems. Comput Methods Appl Mech Eng 357:112552. https://doi.org/10.1016/j.cma.2019.07.021

Plocher J, Panesar A (2019) Review on design and structural optimisation in additive manufacturing: towards nextgeneration lightweight structures. Mater Des 183:108164. https://doi.org/10.1016/j.matdes.2019.108164

Podestá J, Méndez C, Toro S, Huespe A (2019) Symmetry considerations for topology design in the elastic inverse homogenization problem. J Mech Phys Solids 128:54-78. https://doi.org/10.1016/j.jmps.2019.03.018

Prager W, Rozvany G (1977) Optimization of structural geometry. In: Dynamical Systems, Elsevier, pp 265-293. https://doi.org/10.1016/b978-0-12-083750-2.50023-0

Radman A, Huang X, Xie Y (2013a) Topological optimization for the design of microstructures of isotropic cellular materials. Eng Optim 45(11):1331-1348. https://doi.org/10.1080/0305215X. 2012.737781

Radman A, Huang X, Xie YM (2013b) Topology optimization of functionally graded cellular materials. J Mater Sci 48(4):15031510. https://doi.org/10.1007/s10853-012-6905-1

Rodrigues H, Guedes J, Bendsøe M (2002) Hierarchical optimization of material and structure. Struct Multidiscipl Optim 24(1):1-10. https://doi.org/10.1007/s00158-002-0209-z

Rossow M, Taylor J (1973) A finite element method for the optimal design of variable thickness sheets. AIAA J 11(11):1566-1569. https://doi.org/10.2514/3.50631

Schmidt MP, Pedersen CB, Gout C (2019) On structural topology optimization using graded porosity control. Struct Multidiscip Optim 60(4):1437-1453. https://doi.org/10.1007/s00158-019-02275-x

Schury F, Stingl M, Wein F (2012) Efficient two-scale optimization of manufacturable graded structures. SIAM J Sci Comput 34(6):B711-B733. https://doi.org/10.1137/110850335

Sigmund O (1994) Materials with prescribed constitutive parameters: an inverse homogenization problem. Int $\mathrm{J}$ Solids Struct 31(17):2313-2329. https://doi.org/10.1016/0020-7683(94) 90154-6

Sigmund O (1999) On the optimality of bone microstructure. In: Bendsøe MP, Pedersen P (eds) IUTAM Symposium on synthesis in bio solid mechanics. Springer, Netherlands, Dordrecht, pp 221234

Sigmund O (2000) A new class of extremal composites. J Mech Phys Solids 48(2):397-428. https://doi.org/10.1016/S0022-5096(99) 00034-4

Sigmund O (2001) A 99 line topology optimization code written in Matlab. Struct Multidiscipl Opti 21(2):120-127. https://doi.org/10.1007/s001580050176

Sigmund O (2011) On the usefulness of non-gradient approaches in topology optimization. Struct Multidiscip Optim 43(5):589-596. https://doi.org/10.1007/s00158-011-0638-7

Sigmund O, Jensen JS (2003) Systematic design of phononic band-gap materials and structures by topology optimization. Philosophical transactions of the royal society of london series a: mathematical. Phys Eng Sci 361(1806):1001-1019. https://doi.org/10.1098/rsta.2003.1177

Sigmund O, Maute K (2013) Topology optimization approaches. Struct Multidiscip Optim 48(6):1031-1055. https://doi.org/10.1007/s00158-013-0978-6

Sigmund O, Torquato S (1996) Composites with extremal thermal expansion coefficients. Appl Phys Lett 69(21):3203-3205. https://doi.org/10.1063/1.117961

Sigmund O, Aage N, Andreassen E (2016) On the (non-)optimality of michell structures. Struct Multidiscip Optim 54(2):361-373. https://doi.org/10.1007/s00158-016-1420-7

Sivapuram R, Dunning PD, Kim HA (2016) Simultaneous material and structural optimization by multiscale topology 
optimization. Struct Multidiscip Optim 54(5):1267-1281. https://doi.org/10.1007/s00158-016-1519-x

Soto CA, Díaz AR (1993) On the modelling of ribbed plates for shape optimization. Struct Optim 6(3):175-188. https://doi.org/10.1007/bf01743510

Stegmann J, Lund E (2005) Discrete material optimization of general composite shell structures. Int J Numer Methods Eng 62(14):2009-2027. https://doi.org/10.1002/nme.1259

Stromberg LL, Beghini A, Baker WF, Paulino GH (2011) Application of layout and topology optimization using pattern gradation for the conceptual design of buildings. Struct Multidiscip Optim 43(2):165-180. https://doi.org/10.1007/s00158-010-0563-1

Stutz FC, Groen JP, Sigmund o, Bærentzen JA (2020) Singularity aware de-homogenization for high-resolution topology optimized structures. Structural and Multidisciplinary Optimization. https://doi.org/10.1007/s00158-020-02681-6

Tamijani AY, Velasco SP, Alacoque L (2020) Topological and morphological design of additively-manufacturable spatiallyvarying periodic cellular solids. Mater Des 196:109155. https://doi.org/10.1016/j.matdes.2020.109155

Thompson MK, Moroni G, Vaneker T, Fadel G, Campbell RI, Gibson I, Bernard A, Schulz J, Graf P, Ahuja B, Martina F (2016) Design for additive manufacturing: trends, opportunities, considerations, and constraints. CIRP Ann 65(2):737-760. https://doi.org/10.1016/j.cirp.2016.05.004

Thomsen CR, Wang F, Sigmund O (2018) Buckling strength topology optimization of $2 \mathrm{~d}$ periodic materials based on linearized bifurcation analysis. Comput Methods Appl Mech Eng 339:115136. https://doi.org/10.1016/j.cma.2018.04.031

Torquato S, Hyun S, Donev A (2002) Multifunctional composites: optimizing microstructures for simultaneous transport of heat and electricity. Phys Rev Lett 89:266601. https://doi.org/10.1103/PhysRevLett.89.266601

Träff E, Sigmund O, Groen JP (2019) Simple single-scale microstructures based on optimal rank-3 laminates. Struct Multidiscip Optim 59(4):1021-1031. https://doi.org/10.1007/s00158-018-2180-3

Wang C, Zhu JH, Zhang WH, Li SY, Kong J (2018) Concurrent topology optimization design of structures and non-uniform parameterized lattice microstructures. Struct Multidiscip Optim 58(1):35-50. https://doi.org/10.1007/s00158-018-2009-0

Wang C, Gu X, Zhu J, Zhou H, Li S, Zhang W (2020) Concurrent design of hierarchical structures with threedimensional parameterized lattice microstructures for additive manufacturing. Struct Multidiscip Optim 61(3):869-894. https://doi.org/10.1007/s00158-019-02408-2

Wang F, Sigmund O (2020) Numerical investigation of stiffness and buckling response of simple and optimized infill structures. Struct Multidiscip Optim 61(6):2629-2639. https://doi.org/10.1007/ s00158-020-02525-3

Wang F, Lazarov BS, Sigmund O (2011) On projection methods, convergence and robust formulations in topology optimization. Struct Multidiscip Optim 43(6):767-784. https://doi.org/10.1007/s00158-010-0602-y

Wang MY, Wang X, Guo D (2003) A level set method for structural topology optimization. Comput Methods Appl Mech Eng 192(1):227-246. https://doi.org/10.1016/S0045-7825(02)00559-5

Wang X, Xu S, Zhou S, Xu W, Leary M, Choong P, Qian M, Brandt M, Xie YM (2016) Topological design and additive manufacturing of porous metals for bone scaffolds and orthopaedic implants: a review. Biomaterials 83:127-141. https://doi.org/10.1016/j.biomaterials.2016.01.012

Wang Y, Chen F, Wang MY (2017) Concurrent design with connectable graded microstructures. Comput Methods Appl Mech Eng 317:84-101. https://doi.org/10.1016/j.cma.2016.12.007
Wang Y, Groen JP, Sigmund O (2019) Simple optimal lattice structures for arbitrary loadings. Extreme Mech Lett 29:100447. https://doi.org/10.1016/j.eml.2019.03.004

Watts S, Arrighi W, Kudo J, Tortorelli DA, White DA (2019) Simple, accurate surrogate models of the elastic response of threedimensional open truss micro-architectures with applications to multiscale topology design. Struct Multidiscip Optim 60(5):18871920. https://doi.org/10.1007/s00158-019-02297-5

White DA, Arrighi WJ, Kudo J, Watts SE (2019) Multiscale topology optimization using neural network surrogate models. Comput Methods Appl Mech Eng 346:1118-1135. https://doi.org/10.1016/j.cma.2018.09.007

Wu J (2018) Continuous optimization of adaptive quadtree structures. Comput Aided Des 102:72-82. https://doi.org/10.1016/j.cad.20 18.04.008, proceeding of SPM 2018 Symposium

Wu J, Dick C, Westermann R (2016a) A system for high-resolution topology optimization. IEEE Trans Vis Comput Gr 22(3):11951208. https://doi.org/10.1109/TVCG.2015.2502588

Wu J, Wang CC, Zhang X, Westermann R (2016b) Self-supporting rhombic infill structures for additive manufacturing. Comput Aided Des 80:32-42. https://doi.org/10.1016/j.cad.2016.07.006

Wu J, Clausen A, Sigmund O (2017) Minimum compliance topology optimization of shell-infill composites for additive manufacturing. Comput Methods Appl Mech Eng 326:358-375. https://doi.org/10.1016/j.cma.2017.08.018

Wu J, Aage N, Westermann R, Sigmund O (2018) Infill optimization for additive manufacturing - approaching bone-like porous structures. IEEE Trans Vis Comput Graph 24(2):1127-1140. https://doi.org/10.1109/TVCG.2017.2655523

Wu J, Wang W, Gao X (2021) Design and optimization of conforming lattice structures. IEEE Trans Vis Comput Graph 27(1):43-56. https://doi.org/10.1109/TVCG.2019.2938946

Wu K, Sigmund O, Du J (2020) Design of metamaterial mechanisms using robust topology optimization and variable linking scheme. Structural and Multidisciplinary Optimization. https://doi.org/10.1007/s00158-020-02791-1 Accepted

Wu Z, Xia L, Wang S, Shi T (2019) Topology optimization of hierarchical lattice structures with substructuring. Comput Methods Appl Mech Eng 345:602-617. https://doi.org/10.1016/j.cma.2018.11.003

Xia L, Breitkopf P (2014) Concurrent topology optimization design of material and structure within $\mathrm{FE}^{2}$ nonlinear multiscale analysis framework. Comput Methods Appl Mech Eng 278:524-542. https://doi.org/10.1016/j.cma.2014.05.022

Xia L, Breitkopf P (2015) Design of materials using topology optimization and energy-based homogenization approach in Matlab. Struct Multidiscipl Optim 52(6):1229-1241. https://doi.org/10.1007/s00158-015-1294-0

Xie Y, Steven G (1993) A simple evolutionary procedure for structural optimization. Comput Struct 49(5):885-896. https://doi.org/10.1016/0045-7949(93)90035-C

$\mathrm{Xu}$ S, Cai Y, Cheng G (2010) Volume preserving nonlinear density filter based on heaviside functions. Struct Multidiscip Optim 41(4):495-505. https://doi.org/10.1007/s00158-009-0452-7

Xue D, Zhu Y, Guo X (2020) Generation of smoothlyvarying infill configurations from a continuous menu of cell patterns and the asymptotic analysis of its mechanical behaviour. Comput Methods Appl Mech Eng 366:113037. https://doi.org/10.1016/j.cma.2020.113037

Yan S, Wang F, Sigmund O (2018) On the non-optimality of tree structures for heat conduction. Int J Heat Mass Transfer 122:660 680. https://doi.org/10.1016/j.ijheatmasstransfer.2018.01.114

Yang R, Chahande A (1995) Automotive applications of topology optimization. Struct Optim 9(3-4):245-249. https://doi.org/10.1007/BF01743977 
Yu H, Huang J, Zou B, Shao W, Liu J (2020) Stressconstrained shell-lattice infill structural optimisation for additive manufacturing. Virtual Phys Prototyp 15(1):35-48. https://doi.org/10.1080/17452759.2019.1647488

Yuge K, Kikuchi N (1995) Optimization of a frame structure subjected to a plastic deformation. Struct Optim 10(3-4):197-208. https://doi.org/10.1007/BF01742592

Zhang W, Sun S (2006) Scale-related topology optimization of cellular materials and structures. Int J Numer Methods Eng 68(9):9931011. https://doi.org/10.1002/nme.1743

Zhang P, Liu J, To AC (2017) Role of anisotropic properties on topology optimization of additive manufactured load bearing structures. Scr Mater 135:148-152. https://doi.org/10.1016/j.scriptamat.2016. 10.021

Zhang Y, Xiao M, Li H, Gao L, Chu S (2018) Multiscale concurrent topology optimization for cellular structures with multiple microstructures based on ordered SIMP interpolation. Comput Mater Sci 155:74-91. https://doi.org/10.1016/j.commatsci.2018.08.030

Zheng X, Smith W, Jackson J, Moran B, Cui H, Chen D, Ye J, Fang N, Rodriguez N, Weisgraber $\mathrm{T}$ et al (2016) Multiscale metallic metamaterials. Nat Mater 15(10):1100-1106. https://doi.org/10.1038/nmat4694

Zhou M, Rozvany G (1991) The COC algorithm, part II: topological, geometrical and generalized shape optimization. Comput Methods
Appl Mech Eng 89(1):309-336. https://doi.org/10.1016/00457825(91)90046-9

Zhou S, Li Q (2008) Design of graded two-phase microstructures for tailored elasticity gradients. J Mater Sci 43(15):5157-5167. https://doi.org/10.1007/s10853-008-2722-y

Zhou XY, Du Z, Kim HA (2019) A level set shape metamorphosis with mechanical constraints for geometrically graded microstructures. Struct Multidiscip Optim 60(1):1-16. https://doi.org/10.1007/s00158-019-02293-9

Zhu B, Skouras M, Chen D, Matusik W (2017) Two-scale topology optimization with microstructures. ACM Trans Graph 36(5):16. https://doi.org/10.1145/3095815

Zhu JH, Zhang WH, Xia L (2016) Topology optimization in aircraft and aerospace structures design. Arch Computl Methods Eng 23(4):595-622. https://doi.org/10.1007/s11831-015-9151-2

Zhu Y, Li S, Du Z, Liu C, Guo X, Zhang W (2019) A novel asymptoticanalysis-based homogenisation approach towards fast design of infill graded microstructures. J Mech Phys Solids 124:612-633. https://doi.org/10.1016/j.jmps.2018.11.008

Zuo W, Saitou K (2017) Multi-material topology optimization using ordered SIMP interpolation. Struct Multidiscip Optim 55(2):477491. https://doi.org/10.1007/s00158-016-1513-3

Publisher's note Springer Nature remains neutral with regard to jurisdictional claims in published maps and institutional affiliations. 